\title{
Transcranial Direct Current Stimulation of Frontal Cortex Decreases Performance on the WAIS-IV Intelligence Test
}

Kristin K. Sellers ${ }^{a, b}$, Juliann M. Mellin ${ }^{a}$, Caroline M. Lustenberger ${ }^{a}$, Michael R. Boyle ${ }^{a, c}$, Won Hee Lee ${ }^{d}$, Angel V. Petercheve ${ }^{\mathrm{e}, \mathrm{f}, \mathrm{g}}$, and Flavio Frohlich ${ }^{\mathrm{a}, \mathrm{b}, \mathrm{c}, \mathrm{h}, \mathrm{i}, \mathrm{j}}$

a Department of Psychiatry, University of North Carolina at Chapel Hill, Chapel Hill NC 27599 b Neurobiology Curriculum, University of North Carolina at Chapel Hill, Chapel Hill NC 27599 c Department of Biomedical Engineering, University of North Carolina at Chapel Hill, Chapel Hill NC 27599

d Department of Psychiatry, Icahn School of Medicine at Mount Sinai, New York NY 10029 e Department of Psychiatry and Behavioral Sciences, Duke University, Durham NC 27710 f Department of Biomedical Engineering, Duke University, Durham NC 27710 g Department of Electrical and Computer Engineering, Duke University, Durham NC 27710 h Department of Cell Biology and Physiology, University of North Carolina at Chapel Hill, Chapel Hill NC 27599

i Department of Neurology, University of North Carolina at Chapel Hill, Chapel Hill NC 27599

j Neuroscience Center, University of North Carolina at Chapel Hill, Chapel Hill NC 27599

Correspondence should be addressed to: Flavio Frohlich, 115 Mason Farm Rd. NRB 4109F, Chapel Hill, NC. 27599. Phone: 1.919.966.4584. Email: flavio_frohlich@med.unc.edu

The authors declare no competing financial interests. 
Transcranial direct current stimulation (tDCS) modulates excitability of motor cortex. However, there is conflicting evidence about the efficacy of this non-invasive brain stimulation modality to modulate performance on cognitive tasks. Previous work has tested the effect of tDCS on specific facets of cognition and executive processing. However, no randomized, double-blind, sham-controlled study has looked at the effects of tDCS on a comprehensive battery of cognitive processes. The objective of this study was to test if tDCS had an effect on performance on a comprehensive assay of cognitive processes, a standardized intelligence quotient (IQ) test. The study consisted of two substudies and followed a double-blind, between-subjects, sham-controlled design. In total, 41 healthy adult participants completed the Wechsler Adult Intelligence Scale, Fourth Edition (WAIS-IV) as a baseline measure. At least one week later, participants in substudy 1 received either bilateral tDCS (anodes over both F4 and F3, cathode over Cz, $2 \mathrm{~mA}$ at each anode for 20 minutes) or active sham tDCS ( $2 \mathrm{~mA}$ for 40 seconds), and participants in substudy 2 received either right or left tDCS (anode over either F4 or F3, cathode over Cz, $2 \mathrm{~mA}$ for 20 minutes). In both studies, the WAIS-IV was immediately administered following stimulation to assess for performance differences induced by bilateral and unilateral tDCS. Compared to sham stimulation, right, left, and bilateral tDCS reduced improvement between sessions on Full Scale IQ and the Perceptual Reasoning Index. This demonstration that frontal tDCS selectively degraded improvement on specific metrics of the WAIS-IV raises important questions about the often proposed role of tDCS in cognitive enhancement.

\footnotetext{
${ }^{1}$ Abbreviations: Prefrontal cortex (PFC), transcranial direct current stimulation (tDCS), intelligence quotient (IQ), Wechsler Adult Intelligence Scale, Fourth Edition (WAIS-IV), full scale IQ (FSIQ), Verbal Comprehension Index (VCI), Perceptual Reasoning Index (PRI), Working Memory Index (WMI), Processing Speed Index (PSI), transcranial alternating current stimulation (tACS)
} 
Keywords: tDCS, WAIS-IV, IQ, perceptual reasoning, intelligence, brain stimulation

\section{Introduction}

The importance of frontal brain regions has been demonstrated for numerous cognitive processes contributing to intelligence. Dorsolateral prefrontal cortex (DLPFC), a functional area in frontal cortex, is recruited during tests of general intelligence [1-4]. The middle frontal gyrus (the anatomical location of DLPFC) has been implicated in abstracting and integrating logical relationships [5], the ability to resolve interference efficiently [6], and visuospatial reasoning [7]. Medial of the middle frontal gyrus lies the superior frontal gyrus; the lateral part of the superior frontal gyrus has been implicated in aspects of fluid intelligence $[8,9]$, while the medial portion contributes to the default mode network and exhibits deactivation and reduced blood flow during cognitive processing $[10,11]$. Patients with lesions to left superior frontal gyrus demonstrate deficits in working memory compared to controls, particularly in the spatial domain [12]. The most anterior portion of the frontal cortex, prefrontal cortex (PFC), is activated in a performance-dependent way during reasoning and novel problem-solving tests of fluid intelligence [13]. Spatial and verbal tasks requiring high general intelligence differentially increased activation of lateral PFC in comparison to control tasks [3].

Given the widespread involvement of frontal brain areas in higher-order cognitive processing, they represent an attractive target for modulating cognitive function. The ability to both improve cognitive performance in healthy individuals and to alleviate deficits in patients with neuropsychiatric illnesses is the goal of substantial research efforts. A growing body of work has been conducted using transcranial direct current stimulation (tDCS), a form of non-invasive brain stimulation, in an attempt to modulate cognitive abilities [14-16]. Anodal tDCS increases neural activity by depolarizing cortical neurons, whereas cathodal tDCS reduces neural activity by hyperpolarizing neurons [17, 18]. Large neuronal networks are sensitive to such weak perturbations of neuronal membrane voltage caused by 
these electric fields [19-23]. Changes in neuronal excitability induced by tDCS outlast the duration of the stimulation [18], likely through the recruitment of BDNF-dependent plasticity [24-26].

The reported effects of tDCS on cognitive abilities are diverse, with seemingly conflicting reports of increased and decreased performance. The majority of studies conducted to date only used one behavioral assay to test a specific facet of cognitive processing. To our knowledge, no one study has conducted a comprehensive battery of cognitive testing with the same study population in order to assess the effects of tDCS on performance. Thus, we here asked if tDCS affects performance on a comprehensive assay of overall cognition, a standardized Intelligence Quotient (IQ) test. One of the most widely utilized IQ tests is the Wechsler Adult Intelligence Scale, Fourth Edition (WAIS-IV). Use of the WAIS-IV test is advantageous because separate index scores can be calculated to provide insight into more fine-grained components of intelligence. Previous work has suggested that the different aspects of intelligence probed by the WAIS-IV indices and subtests do not share a single common neuronal substrate [27].

Because of the broad activation of frontal areas, we first tested if bilateral tDCS over DLPFC changed performance on the WAIS-IV. We hypothesized that by targeting frontal areas with tDCS, we would induce improved performance. Interestingly, the effects of stimulation were detrimental to IQ, specifically in tasks of perceptual reasoning. We then conducted a second study to test the effects of unilateral right or left tDCS on performance on the WAIS-IV; similar performance decreases were found with additional evidence for more pronounced decreases for right tDCS. 


\section{Materials and Methods}

\subsection{Participants.}

In total, 44 healthy adults were recruited for this study (21 males, 23 females, mean age $=22.1$ years, $S D=4.72$ years) from the University of North Carolina at Chapel Hill community. The study was divided into two consecutive substudies for which participants were recruited separately. For Substudy 1, 22 participants participated in Session 1 of IQ testing, and 21 of these participants returned for Session 2 and received either bilateral tDCS or sham tDCS with subsequent repeat IQ testing. One participant could not be contacted for Session 2 and was therefore excluded from the study. For Substudy 2, 22 participants completed Session 1 of IQ testing, and 20 of these participants returned for Session 2 and received either right tDCS (anodal electrode on right hemisphere) or left tDCS (anodal electrode on left hemisphere) with subsequent repeat IQ testing. Analysis was conducted on the 20 participants who completed both sessions. No participants took part in both Substudy 1 and Substudy 2 . By self-report, participants did not have a history of neurologic or psychiatric illness, were not currently using medication for a neurologic or psychiatric illness, were not currently undergoing counseling or psychotherapy treatment, did not have a first degree relative with a neurologic or psychiatric condition, had never undergone brain surgery, had no brain devices/implants, did not have any cardiovascular diseases, and were not pregnant. All participants signed written consent prior to participation. This study was approved by the UNC - Chapel Hill IRB.

\subsection{Experimental Design}

Both Substudy 1 and Substudy 2 followed a double-blind, between-subjects design with repeated-measure testing of IQ. In both substudies, participants completed the full WAIS-IV (Pearson Education, Inc., San Antonio, TX), as detailed below during the initial study visit (Session 1, baseline). Participants returned at least one week later (Session 2, mean time between sessions $=23.6$ days, SD $=$ 
19.7) and received either sham or bilateral tDCS (Substudy 1, Figure 3A) or right or left tDCS (Substudy 2, Figure 3B) and immediately afterwards completed the same WAIS-IV test. At the conclusion of the Session 2, participants completed a questionnaire asking if they believed they received stimulation.

\subsection{Wechsler Adult Intelligence Scale, Fourth Edition (WAIS-IV).}

The WAIS-IV is a comprehensive clinical instrument for assessing intelligence of adults between the ages of $16-90$ years. There has been substantial demonstration of the test's validity and reliability. The test is composed of 15 core and supplemental subtests which contribute to a composite score that represents general intellectual ability (full scale IQ, FSIQ) and scores in indices of specific cognitive areas. While the FSIQ is considered the best measure of overall cognitive ability, the test issuer recommends to further report the index scales that all contribute to the FSIQ: Verbal Comprehension Index (VCI), Perceptual Reasoning Index (PRI), Working Memory Index (WMI), and Processing Speed Index (PSI). The $\mathrm{VCl}$ measures verbal reasoning, verbal concept formation, and knowledge acquired from the environment [28]. Strategies to solve the problems presented in this index may also utilize nonverbal factors such as forming mental pictures. The PRI measures perceptual and fluid reasoning, spatial processing, and visual-motor integration [29]. The WMI measures working memory, the ability to temporarily hold information in memory, manipulate or perform a mental operation on this information, and produce a response [29]; these processes require attention, concentration, mental control, and reasoning, and have been shown to be an essential component of higher order cognitive processes [30-32]. The PSI provides a metric of the participant's ability to quickly and correctly scan, sequence, or discriminate simple visual information [29]; this measures incorporates short-term visual memory, attention, and visual-motor coordination [33-35]. Important to note, the PSI includes cognitive decision-making or learning components, and is not simply measuring reaction time or visual discrimination. 
These index scales are further composed of core and supplemental subtests as described in Table $1[29,36]$. The raw scores from these subtests are scaled to a metric with a mean of 10 and a standard deviation of 3, based on the given age group. Different scales contribute to standard composite scores (i.e. VCI, PRI, WMI, PSI, and FSIQ), metrics with mean of 100 and standard deviation of 15. For our application, these metrics may be useful in isolating which facet(s) of intelligence are modulated by tDCS. All general testing, administration, and scoring guidelines were followed as prescribed by Pearson Education, Inc [37].

\subsection{Transcranial direct current stimulation.}

For all participants, two stimulation electrodes $(5 \times 7 \mathrm{~cm}$, placed in saline soaked sponge sleeves) were positioned bilaterally over the middle frontal gyri, at positions F4 and F3 of the International 10-20 System. An additional single electrode located over $\mathrm{Cz}$ served as the cathode for both stimulation sites (Figure 1A). For bilateral stimulation (Substudy 1), two simultaneously triggered single channel stimulators were used to administer anodal tDCS to the frontal electrodes (NeuroConn DC-STIMULATOR PLUS, NeuroConn Ltd., Ilmenau, Germany). Both the participant and the experimenter administering stimulation and the WAIS-IV were blind to the stimulation condition until completion of the study. Bilateral stimulation consisted of 20 minutes of $2 \mathrm{~mA}$ direct current applied to each of the frontal electrodes (Figure 1B, anodal current density at F3 and F4 $=0.057 \mathrm{~mA} / \mathrm{cm}^{2}$, cathodal current density at $\left.\mathrm{Cz}=0.114 \mathrm{~mA} / \mathrm{cm}^{2}\right)$. Sham stimulation consisted of 40 seconds stimulation at $2 \mathrm{~mA}$ in the same electrode configuration as for the bilateral stimulation, to mimic the skin sensation of bilateral stimulation (Figure 1C). Stimulation occurred while participants were resting but awake, sitting comfortably with eyes open. In Substudy 2, right tDCS or left tDCS was only delivered to either F4 or F3 (current density at F4, F3, and $\left.\mathrm{Cz}=0.057 \mathrm{~mA} / \mathrm{cm}^{2}\right)$, with the same duration and amplitude as used for Substudy 1 . Sham stimulation (40 seconds) was delivered to the non-targeted hemisphere (Figures 1D-E). The current density and duration 
used in this study are well within currently accepted safety guidelines for tDCS [38]. We adopted a study design that avoided stimulation during test performance since WAIS-IV test duration exceeds the maximal stimulation duration permitted by recent tDCS safety guidelines [39].

\section{5 tDCS electric field modeling}

To determine which cortical structures were targeted by our stimulation paradigm, we simulated the electric field generated by the tDCS electrode configuration using a previously developed realistic finite element model of a human head incorporating heterogeneous and anisotropic tissue conductivity $[40,41]$. The head model is of a single subject (34 year old male) who did not participate in the study. Nevertheless, the simulated electric field distribution is informative of the general properties of the tDCS electrode configuration and current strength used in this study. The modeling procedure is briefly summarized below.

The model was derived from structural T1-weighted MRI images $\left(1 \times 1 \times 1 \mathrm{~mm}^{3}\right.$ voxel). Image preprocessing included AC-PC spatial alignment, bias field correction, anisotropic diffusion filtering, and skull stripping [40]. Individual tissue probability maps corresponding to gray matter, white matter, and cerebrospinal fluid (CSF) were automatically created using the segmentation tool FAST in FSL (FMRIB Analysis Group, Oxford, UK) [42]. The non-brain regions were manually segmented into 11 tissue compartments including skin, muscle, skull compacta, skull spongiosa, vertebrae, spinal cord, lens, eyeball, sclera, optic nerve, and sinus, using a combination of segmentation editing tools from ITK-SNAP [43] and an in-house segmentation algorithm based on thresholding and mathematical morphological operations. We modeled the tDCS sponge electrodes as rectangular cuboids with $5 \mathrm{~cm} \times 7 \mathrm{~cm}$ surface intersecting the head (Figure 2). The complete 3D head model incorporating the tDCS electrodes was adaptively tessellated to produce the finite element model using the restricted Delaunay triangulation algorithm [44]. The electrical conductivity of the head tissues was assigned as in [41]. The electrodes 
were assumed to have the conductivity of saline $(1.4 \mathrm{~S} / \mathrm{m})$. Constant electric current was applied to the electrode surfaces away from the head. For substudy 1, $2 \mathrm{~mA}$ were applied to each of the frontal electrodes and $-4 \mathrm{~mA}$ to the posterior cathode. For substudy 2, $2 \mathrm{~mA}$ were applied to the frontal electrode (right and left tDCS modeled separately), $-2 \mathrm{~mA}$ to the posterior cathode, and $0 \mathrm{~mA}$ to the frontal electrode contralateral to the stimulated side. Finally, the electric field was computed by solving the Laplace equation using the preconditioned conjugate solver within ANSYS (ANSYS Inc., Canonsburg, PA, USA) [40].

\subsection{Data Analysis.}

For each administration of the WAIS-IV, raw scores were calculated for each of the 15 subtests. Age-normalized scaled scores were then determined from these raw scores in accordance with scoring guidelines provided by Pearson Testing, Inc. The scaled scores were tallied to provide sums of the scaled scores, and converted to composite scores: FSIQ, VCl, PRI, WMI, and PSI.

\subsection{Statistical Analysis.}

Custom-written scripts in MATLAB (Mathworks, Natick, MA) and R (R Foundation for Statistical Computing, Vienna, Austria) [45] were used for analysis. Libraries used in R included Ime4 [46] and pbkrtest [47]. Wilcoxon rank-sum test was used to determine if continuous variables (age, time of day for Session 1, and time of day for Session 2) differed by stimulation condition (sham, right, left, or bilateral tDCS); chi-squared test was used to determine if categorical variables (gender) differed between stimulation conditions, and whether perception of stimulation differed between stimulation conditions.

We performed a linear mixed model analysis of the relationship between scores on the WAIS-IV and stimulation condition. We entered stimulation condition (sham, right, left, or bilateral tDCS) and 
session (baseline or post stimulation [post-stim]) as fixed effects, and subjects as a random effect into the model. Visual inspection of the residual plots did not reveal any obvious deviations from normality or homoscedasticity. We used the Kenward-Roger approximation to perform F-tests and to estimate $p$ values for each factor and their interaction in the mixed model [48]. In the case of significant or trend level interactions, we conducted post-hoc Welch's t-tests in order to determine the source of significance. Specifically, we compared scores between stimulation conditions within each session, and then calculated the difference in scores across sessions for each stimulation condition. Significance was determined by $p<0.05$, and trend by $p<0.1$. We present both raw $p$-values and $p$-values corrected for multiple comparisons using False Discovery Rate (FDR) calculations. Unless otherwise stated, bar graphs depict the mean change in score \pm sem. For each participant, the change in score was calculated between sessions (session 2 - session 1); these values were then averaged across participants.

Spearman's rank-order correlation was used to assess if the effect of stimulation was related to subtest administration order; group means of the change in score from Session 1 to Session 2 for each subtest were tested for significant correlation with subtest order (1 to 15). Spearman's rank-order correlation was also used to assess for age-dependent effects of stimulation; change in score between Session 1 and Session 2 on each WAIS-IV metric of interest for each stimulation condition was tested for significant correlation with participant age. 


\section{Results}

The results of both substudies are detailed below. In substudy 1 , we tested the hypothesis that bilateral tDCS applied over frontal regions would improve performance on the WAIS-IV IQ test compared to sham stimulation. To our surprise, we found that bilateral tDCS had a negative effect on test performance compared to sham stimulation. Therefore, we conducted a second substudy in order to test whether the effects of right or left tDCS differed from bilateral stimulation. In agreement with our findings from substudy 1 , we found that both right and left tDCS induced similar reductions in practice gains on the WAIS-IV. We present the data combined across these two substudies for a number of reasons. The WAIS-IV has been specifically designed and validated to produce reliable scores across different test administrators. In our study, there were no significant differences in baseline score between the different stimulation groups for FSIQ or any of the index scores. In addition, the similar finding of both studies (that tDCS decreased practice gains) indicates that this effect is robust.

Therefore, presenting the results of the two substudies combined provides a more comprehensive view of the effects of unilateral and bilateral tDCS on a standardized assessment of IQ.

\subsection{Demographic and individual characteristics.}

Participants in the stimulation groups did not differ significantly in age (sham mean $=25.7$ years, sham $S D=8.41$ years, right mean $=20.6$ years, right $S D=2.12$ years; left mean $=21.5$ years, left $S D=$ 2.27 years; bilateral mean $=20.5$ years, bilateral $S D=2.02$ years, Wilcoxon rank-sum test, all $p>0.1$ ), time of day for Session 1 (Wilcoxon rank-sum test, all $p>0.1$ ), time of day for Session 2 (Wilcoxon ranksum test, all $p>0.1$ ), or gender (sham $=5$ males, 5 females; right $=4$ males, 6 females; left $=6$ males, 4 females; bilateral $=5$ males, 6 females, $\left.\chi^{2}(3, n=41)=0.867, p=0.833\right)$. In substudy $1,57 \%$ of participants correctly guessed whether they received brain stimulation, with chance level at $50 \%$ (possible responses were 'Yes' and 'No')( chi-square test assessing for association between stimulation 
condition and perception of stimulation , $\left.\chi^{2}(1, N=21)=3.82, p=0.051\right)$. In substudy 2 , only $30 \%$ of participants correct guessed their stimulation condition, with change level at 25\% (possible responses were 'Right side of head', 'Left side of head', 'Both sides of head', 'No stimulation')(chi-square test assessing for association between stimulation condition and perception of stimulation $\chi^{2}(1, N=20)=0, p$ $>0.05$ ). In substudy $2,55 \%$ of participants thought stimulation influenced their performance on the IQ test ('Do you think your performance on the IQ test was affected by the transcranial current stimulation').

\subsection{Effects of unilateral and bilateral tDCS on WAIS-IV scores}

In the finite element simulation, our electrode montage predominantly induced electric fields in the middle and superior frontal gyri of frontal cortex (Figure 2). In the case of bilateral stimulation, electric fields were nearly symmetrical in both hemispheres (Figure 2A-D). For left tDCS, the electric field predominantly affected the left hemisphere, with limited spread into the right hemisphere (Figure $2 \mathrm{E}$ G). Similarly, right tDCS targeted right middle and superior frontal gyri with only minimal applied electric field in the left hemisphere (Figure $2 \mathrm{H}-\mathrm{J}$ ). In order to test the effects of tDCS on intelligence, we assessed change in performance on the indices and subtests of the WAIS-IV as a function of stimulation condition. See Table 2 for the group means of scaled composite scores. The FSIQ is a global estimate of an individual's current level of cognitive ability, and is the most reliable and valid estimate of an individual's intellectual ability [36]. In the linear mixed model assessing the effect of stimulation on FSIQ, the factor session was significant $(F(1,40)=100, p<0.001)$, the factor stimulation condition was nonsignificant $(F(3,37)=0.813, p>0.1)$, but the interaction between session and stimulation condition was significant $(F(3,37)=4.38, p=0.00979)$. Post-hoc t-tests revealed that FSIQ did not differ significantly between groups at baseline (all $p>0.1$ ) or after stimulation (all $p>0.1$ ). However, the difference between FSIQ during post-stim and baseline was significantly different between sham and 
unilateral/bilateral stimulation (sham vs right tDCS: $t(17.3)=3.63$, uncorrected $p=0.00204$, corrected $p$ $=0.0079 ;$ sham vs left tDCS: $\mathrm{t}(17.6)=2.28$, uncorrected $\mathrm{p}=0.0352$, corrected $\mathrm{p}=0.0790$; sham vs bilateral tDCS: $\mathrm{t}(18.8)=2.76$, uncorrected $\mathrm{p}=0.0127$, corrected $\mathrm{p}=0.0348)$. Right tDCS, left tDCS, and bilateral tDCS led to reduced practice gains compared to the sham condition (Figure 4: change in scores between session was calculated for each participant, and then averaged across participants, to provide mean difference in $\mathrm{FSIQ} \pm \mathrm{SEM}$, sham $\mathrm{tDCS}=9.80 \pm 0.998$, right $\mathrm{tDCS}=4.10 \pm 1.22$; left $\mathrm{tDCS}=6.30 \pm$ 1.16; bilateral tDCS $=5.55 \pm 1.18$ ). Thus, unilateral and bilateral tDCS significantly reduced the practice gains in FSIQ between testing sessions compared to sham stimulation.

The WAIS also provides index scores to assess specific cognitive areas. To examine which index scale(s) contribute to the tDCS-induced effects on the FSIQ, we further performed linear mixed model analyses for each of the four index scales. The results are summarized in Table 3 and Figure 5. The factor session was significant in all indices except $\mathrm{VCl}$, indicating that performance differed from Session 1 to Session 2 for the PRI, WMI, and PSI. Only VCI revealed a significant effect of the factor stimulation condition. Interestingly, only the PRI showed a significant interaction between session and stimulation condition. Post-hoc testing demonstrated that compared to sham stimulation (mean difference in PRI \pm SEM $=12.1 \pm 2.73$ ), practice gains in the PRI were significantly lower following right tDCS (mean difference in PRI $\pm \mathrm{SEM}=1.90 \pm 20.5, \mathrm{t}(16.7)=2.99$, uncorrected $\mathrm{p}=0.00837$, corrected $\mathrm{p}=0.022)$, lower at trend level following left tDCS (mean difference in PRI \pm SEM $=5.90 \pm 1.82, t(15.7)=1.89$, uncorrected $p=0.0773$, corrected $p=0.135$ ), and lower at trend level following bilateral tDCS (mean difference in $\mathrm{PRI} \pm \mathrm{SEM}=4.64 \pm 2.44, \mathrm{t}(18.51)=2.04$, uncorrected $\mathrm{p}=0.0562$, corrected $\mathrm{p}=0.077$ ) (Figure 6B). This effect was not driven by differences in baseline PRI between the groups (all $p>0.1$ ).

Given the significant and trend-level interactions between session and stimulation condition on the PRI, we next analyzed the three subtests that comprise the PRI (Block Design, Matrix Reasoning, and Visual Puzzles). These subtests were performed consistently in the order prescribed by the WAIS-IV 
(Block Design: $1^{\text {st }}$, Matrix Reasoning: $4^{\text {th }}$, and Visual Puzzles: $8^{\text {th }}$ subtests). Results of the linear mixed model on these subtests are summarized in Table 4 and Figure 6. Block Design and Visual Puzzles provide a significant session effect, while Matrix Reasoning had a trend level effect of session. Visual Puzzles exhibited a significant effect of stimulation condition. For Matrix Reasoning and Visual Puzzles, the interaction between session and stimulation condition was significant or significant at trend level.

Post-hoc t-tests revealed that Matrix Reasoning did not differ significantly between groups at baseline (all p>0.1). However, practice gains were dependent on the stimulation condition (Figure 6, mean difference in Matrix Reasoning \pm SEM: sham tDCS $=1.80 \pm 0.854$, right tDCS $=-0.200 \pm 0.663$, left $\mathrm{tDCS}=1.70 \pm 0.761$, bilateral tDCS $=-0.273 \pm 0.469)$. Specifically, right tDCS decreased the practice gain at trend level compared to the sham condition $(t(17.0)=1.850$, uncorrected $p=0.0818$, corrected $p=$ 0.094); bilateral tDCS also decreased practice gains at the trend level compared to sham tDCS $(t(14.1)=$ 2.128, uncorrected $p=0.0515$, corrected $p=0.094)$; practice gains were smaller following right tDCS compared to left tDCS at the trend level $(\mathrm{t}(17.7)=-1.882$, uncorrected $\mathrm{p}=0.0764$, corrected $\mathrm{p}=0.094)$; and bilateral tDCS resulted in reduced practice gains compared to left tDCS $(t(15.2)=2.21$, uncorrected $p=0.0431$, corrected $p=0.094$ ) (Figure 6B).

In the case of Visual Puzzles, post-hoc t-tests indicated that right and left tDCS significantly decreased practice gains compared to the sham condition (Figure 6C, mean difference in Visual Puzzles \pm SEM: sham tDCS $=2.60 \pm 0.733$, right $\mathrm{tDCS}=0.200 \pm 0.327$, left $\mathrm{tDCS}=0.400 \pm 0.980$, bilateral $\mathrm{tDCS}=$ $1.82 \pm 0.807$. Sham tDCS vs right tDCS: $t(12.4)=2.99$, uncorrected $p=0.0109$, corrected $p=0.15$; sham tDCS vs left tDCS: $t(16.7)=1.80$, uncorrected $p=0.090$, corrected $p=0.15)$. No baseline differences were significant, but left vs bilateral tDCS exhibited trend-level differences at baseline $(t(16.9)=2.05, p=$ 0.0568).

\subsection{WAIS-IV subtest order and participant age do not explain effects of tDCS}


TDCS has been shown to induce outlasting effects on the excitability of motor cortex for multiple hours, assessed by measuring motor evoked potentials induced by transcranial magnetic stimulation pulses [49]. However, no comparable physiological measurement exists to assess outlasting changes in excitability in frontal cortex. In theory, elapsed time since the end of stimulation could affect which subtests showed significant modulation based on stimulation condition. To test for this possibility, we calculated the correlation between group mean of change in score between sessions and subtest administration order. We found no significant correlation between the order of subtests and change in performance between the testing sessions for substudy 1 (Figure 7A: Mean of change in subtest scores, in order of subtest administration. Spearman's correlation of change in score and subtest order was non-significant, sham tDCS: rho $=-0.170, p=0.544$; bilateral tDCS: rho $=-0.186, p=0.506$ ) or substudy 2 (Figure 7B, right tDCS: rho $=0.245, p=0.379$; left tDCS: rho $=0.218, p=0.434$ ).

Lastly, the plasticity recruited by tDCS is likely age-dependent [50]. Therefore, effects of stimulation could be masked by the age of participants. To test for this, we calculated correlations between change in scores in each stimulation group and participant age. Correlations were nonsignificant for all indices of the WAIS-IV and all subtests of the PRI, except for Figure Weights in the left tDCS condition (all p-values $>0.1$, except Figure Weights: left, rho $=0.755, p=0.011$ ). 


\section{Discussion}

In the last decade, numerous studies have investigated whether tDCS can be used to improve cognitive abilities or alleviate deficits associated with neuropsychiatric diseases. The resulting literature is diverse, with reports of improved and/or decreased cognitive performance with stimulation in the domains of working memory, executive functioning, verbal and semantic processing, cognitive control during emotion regulation, verbal tasks, visuospatial memory, word fluency, verbal memory, categorization learning, memory performance and learning, language comprehension, and attention control $[15,16]$. In each of these studies, tDCS was targeted to DLPFC, through electrodes positioned over F4 and F3. Here, we sought to test if unilateral or bilateral tDCS over DLPFC altered performance on a standardized IQ test, a multi-faceted and comprehensive assessment of cognitive abilities. Specifically, the purpose of substudy 1 was to investigate if bilateral tDCS (anodes over both F4 and F3, cathode over $\mathrm{Cz}$ ) modulates performance on a standard IQ test. Based on our finite element modeling, stimulation in this study primarily targeted the middle and superior frontal gyri. Other studies which applied stimulation through anodes positioned at F4 or F3 attributed stimulation effects to DLPFC, a functional region which lies on the middle frontal gyrus.

The application of bilateral stimulation was chosen because IQ tests assess multiple cognitive functions, which utilize broad frontal areas; previous investigations of the effects of brain stimulation on cognitive function have used similar approaches [51]. As a note, previous reports of 'bilateral' tDCS primarily positioned the anode on one side of the head (often M1) and the cathode over the same region on the contralateral side of the head. This is markedly different from our bilateral stimulation, in which both sides of the head received anodal stimulation, and a common cathode was positioned at $\mathrm{Cz}$. In a follow-up study, we tested whether the effects of right or left tDCS differed from bilateral stimulation. We found that all forms of tDCS (right, left, and bilateral) impaired performance on the FSIQ, compared to sham stimulation. More detailed analysis revealed that stimulation induced selective 
impairment of the PRI (significant for right tDCS, trending significant for left and bilateral tDCS). Of the three subtests which contribute to the PRI, performance on two exhibited selective impairment based on tDCS. Specifically, right tDCS decreased performance on Matrix Reasoning at the trend level compared to both sham and left tDCS, while the effects of left tDCS for this subtest were indistinguishable from sham stimulation. For Visual Puzzles, both right and left tDCS reduced practice gains compared to sham stimulation. There were no differences in baseline scores between the stimulation groups, thus this cannot explain the stimulation-induced reduction in practice gains on the FSIQ or PRI. Furthermore, our results from two independent substudies support each other. Data collected during the first substudy demonstrated that bilateral stimulation was detrimental to performance compared to sham stimulation. The results from our second substudy, performed following the completion of substudy 1 , further support this finding and demonstrate that both right and left tDCS reduce practice gains in both the FSIQ and PRI. These results suggest that unilateral and bilateral tDCS over DLPFC impair performance on specific perceptual reasoning tasks, but may not affect verbal comprehension, working memory, or processing speed abilities.

\subsection{Potential mechanisms of tDCS}

The mechanisms underlying the effects of tDCS are still under investigation. An important first consideration is that the physiological underpinnings of tDCS-induced changes differ during stimulation compared to after stimulation. Our discussion will focus on changes following stimulation, according to the experimental paradigm implemented in our study. Neurophysiological, imaging, and pharmacological investigations have demonstrated a number of physiological changes following tDCS $[52,53]$, which are believed to depend upon changes in synaptic strength mediated by NMDA receptors and both GABAergic and glutamatergic synapses [54, 55]. Early work with tDCS conducted in motor cortex demonstrated that anodal stimulation increased excitability while cathodal stimulation inhibited 
activity [18]. However, a meta-analysis looking at cognitive studies indicates that in non-motor areas, anodal stimulation may indeed still increase excitability but cathodal stimulation does not induce inhibitory effects [56]; the authors posited that this may be due to higher brain activation states during cognitive tasks, and the greater range of behavioral measures used in cognitive tasks compared to TMSinduced motor evoked potentials, which are used to measure motor cortex excitability.

Using whole-brain arterial spin labeling, anodal tDCS in left DLPFC has been shown to increase perfusion to brain regions structurally connected with left DLPFC, increase functional coupling between bilateral DLPFC, but decrease functional coupling between left DLPFC and bilateral thalami; immediately following tDCS, perfusion decreased in the frontal lobes bilaterally, in an anatomical distribution similar to that of the default mode network [57]. Contrastingly, fMRI has demonstrated that immediately following anodal tDCS over left DLPFC, the default mode network and bilateral fronto-parietal networks exhibited greater co-activation and connectivity [58]. If perfusion indeed decreases immediately following tDCS, this may mediate the deficits in performance on the IQ test we observed in the present study. Future work will be needed to determine if the brain regions mediating perceptual reasoning abilities may be particularly affected by reduced perfusion, as could be hypothesized based on our finding of selective impairment in the PRI following unilateral and bilateral tDCS.

\section{2 tDCS effects on Perceptual Reasoning}

We found that both unilateral and bilateral tDCS induced selective impairments in the PRI of the WAIS-IV. The PRI measures fluid reasoning, with tasks that assess nonverbal concept formation, visual perception and organization, visual-motor coordination, learning, and the ability to separate figure and ground in visual stimuli. Previous reports have found that anodal tDCS over frontal cortex improves perceptual sensitivity [59], learning to identify concealed objects in naturalistic surroundings [60], and perceptual learning [61]. Other work has applied anodal tDCS to visual areas and improved perceptual 
learning [62], while parietal tDCS has improved reaction time on contralateral search tasks [63].

However, another body of research has found that both right anodal and cathodal tDCS on DLPFC impair the efficiency of managing stimulus-response feature bindings, which taxes perceptual abilities; this

study proposed that tDCS could create reversible 'frontal lesions', for at least specific cognitive tasks [64, 65]. Another study found that anodal and cathodal tDCS over medial-frontal cortex did not change perceptual processing, but only subsequent error- and feedback-related negativities [66]. Furthermore, anodal tDCS of V1 was shown to block overnight consolidation of visual learning [67]. Thus, our work and previous studies indicate that at least some forms of frontal tDCS may produce a 'frontal lesion' effect, in which stimulation impairs performance on facets of perceptual reasoning. Future work will be needed to elucidate if these specific effects results from the location of applied stimulation, the specific tasks being tested, or a combination of these factors.

\subsection{Previous Studies on tDCS and Working Memory}

Our finding that tDCS does not affect working memory performance joins a diverse set of studies investigating the modulation of working memory ability by tDCS. A number of studies have found that anodal tDCS to left DLPFC improves performance on verbal and non-verbal working memory tasks $[68,69]$, with some qualifiers such as improvement measured only in males [70], or that stimulation was beneficial but selectively in older adults with more education [71]. Other reports found no effects of tDCS on working memory accuracy [72-74], but improvement in only reaction time [75-77]. Conversely, one study found that accuracy, but not reaction time, was improved by anodal tDCS applied to DLPFC compared to sham and cathodal stimulation [78]. Additional evidence suggests that left tDCS improves verbal working memory while right tDCS improves visuospatial working memory [79]. There is weak evidence that concurrently administered anodal tDCS to left DLPFC during a working memory task 
may improve subsequent testing on another working memory test, compared to just tDCS or administration of the first working memory task alone [80].

While the conceptualization of working memory is helpful for discussion and study, this remains a broad construct which incorporates multiple cognitive functions (including but not limited to rehearsal, maintenance, updating, and executive function) [32]. Work conducted to disentangle which contributions to working memory may be modulated by tDCS has provided some insight that anodal tDCS over left DLPFC may not improve the ability to overcome bias [81] but may be mediated by specific effects on selective attention because of the presence of interference [82]. Overall, it is still unclear if and how tDCS modulates working memory. A meta-analysis on the effects of tDCS applied to DLPFC on n-back working memory tests (studies published through February 2013) indicates that only reaction time, but not accuracy, is improved by stimulation [83]. Of critical importance for comparison of studies, reaction time is not measured in the WAIS-IV working memory subtest, and thus our results are not directly comparable to tests of working memory which assessed reaction times. We did not conduct MRI scans to accommodate anatomical differences across participants; however, there is evidence that modulation of performance on working memory may result from differing current densities at DLPFC, despite consistent electrode placement according to the 10-20 system across participants [84]. Importantly, our electrode montage differed in the location of the cathode compared to many previous studies. Thus, our induced current is not directly comparable to previous studies which administered anodal tDCS to DPFC.

\subsection{Investigations of tACS and intelligence}

Other studies assessing the role of brain stimulation on forms of intelligence have utilized transcranial alternating current stimulation (tACS) that employs alternating current waveforms. tACS targets the temporal organization of network activity through frequency-specific enhancement of 
cortical oscillations and coherence $[19,85,86]$. Such temporal structure plays an important role in mediating cognitive abilities such as attention [87], working memory [88, 89], and encoding and retrieval of memory $[90,91]$. One study found that gamma frequency tACS administered over left middle frontal gyrus reduced the amount of time required to solve the Raven's matrices [92]. However, there was no difference between tACS and sham groups in accuracy. Another study found that theta frequency tACS over parietal cortex improved performance on a modified version of Raven's progressive matrices, mainly through participants correctly solving more difficult task items [93]. Interestingly, the Raven's matrices in these studies are similar to the Matrix Reasoning subtest of the PRI in the WAIS-IV. The Matrix Reasoning subtest has previously been used as a measure of fluid intelligence [94], and scores on the test are speed-dependent. Thus, tDCS and tACS may have opposite effects on performance on assays of fluid intelligence. This difference likely relates to the mechanistic difference between tDCS (inducing changes in excitability) and tACS (modulating temporal patterning of activity). Future work will be required to more fully understand the role of each of these stimulation modalities on fluid intelligence, as well as other aspects of cognitive processing.

\subsection{Neurobiological Substrate of Intelligence}

The neurobiological substrate of intelligence is still unknown. Historically, it has been posited that the diverse functional roles of DLPFC provide a unified neural architecture for Spearman's classic general $(g)$ factor model of intelligence $[3,4]$. In this theory of intelligence, the $g$ factor posits that an individual's mental performance across a broad range of cognitive tests is often comparable [95]. However, recent work utilizing lesion mapping has demonstrated that performance on metrics used to measure the $g$ factor of intelligence depend upon fronto-parietal networks, in accordance with the Parieto-Frontal Integration Theory (P-FIT) of intelligence [96, 97](however, results from [97] also demonstrate that regions of frontopolar cortex may play a unique role in $g$ ). In the P-FIT 
conceptualization of intelligence, different cognitive functions are mediated by a broadly distributed network of functionally specialized brain regions, including prefrontal, parietal, occipital, and temporal association cortices [27, 98-100]. Four stages of information processing are supported by critical information flow between multiple brain regions, in particular frontal and parietal regions [101]. In agreement of this model, the neurobiological substrate of intelligence has been hypothesized to correspond to genetically determined brain structure and connectivity [102-107], and individuals with more efficient whole brain network organization have a higher overall level of intelligence [108-110]. Thus, while our study was not a direct assay of the neurobiological substrate of intelligence, our results may provide insight on this question. The differential effects of tDCS on WAIS-IV index and subtest performance is in agreement with P-FIT; because of the spatial distribution of brain structures implicated in the variety of cognitive processes included in the WAIS-IV, the stimulation would differentially affect these networks.

\subsection{Implications for Society: DIY Stimulation}

The complexity of the neurobiological substrates of intelligence is particularly relevant given the growing interest of the lay public in brain stimulation. In a simplified form, many people believe that increased excitability induced by brain stimulation can be performance-enhancing (motivated by communication of findings that cognitive training enhances brain activity measured by fMRI in prefrontal and parietal area, including the middle frontal gyrus [111]). The relative low cost, technical ease, and attractive hypothetic benefits of brain stimulation have sparked the development of commercially available do-it-yourself (DIY) brain stimulation devices. However, DIY devices are not validated and may not be safe [112]. Furthermore, our study casts doubt on the hype surrounding the simplified idea that applying brain stimulation will lead to better cognitive performance [113]. In fact, the opposite effect was demonstrated in our study. This finding together with the very real safety risks 
of DIY brain stimulation will hopefully discourage the wider, uncontrolled use of tDCS outside the research laboratory.

\subsection{Limitations and Conclusions}

There are certain limitations to this study which are important to consider. Because we wanted to administer the full version of the WAIS-IV in accordance to standard testing protocols, the same test was administered to each participant twice. It was clear that participants exhibited a practice effect as a result of the retesting [114]. However, as all participants in all stimulation groups underwent this same procedure, we do not anticipate the reported effects to depend upon differences induced by this retesting. However, we cannot fully exclude the possibility that unilateral or bilateral tDCS induced a difference in recall from Session 1, rather than a true alteration to the neural substrates underlying cognitive processing. However, in such a case we would expect performance modulation that is less task-specific than what we found, since not only the perceptual reasoning index showed a learning effect for the sham group. Another limitation to keep in mind is that tDCS was administered before participants completed the WAIS-IV during Session 2. Thus, elapsed time since the end of stimulation might affect which subtests showed significant modulation. However, our correlational analysis showed that time of test was not associated with tDCS-induced performance changes and could therefore not explain the specific stimulation effect on Matrix Reasoning or Visual Puzzles. Lastly, there is growing recognition of important considerations when using tDCS in cognitive research, such as participant motivation [115]; our study may have suffered from one of these problems.

In conclusion, we found that unilateral and bilateral tDCS over DLPFC reduced practice gains in a comprehensive test of intelligence, with selective impairment in perceptual reasoning. The impairment found here suggests that tDCS indeed targeted selective neuronal network dynamics that enable cognition. Our study highlights that increasing neuronal activity in some frontal areas may not be 
beneficial to cognitive processing, with additional evidence that the timing of tDCS relative to task performance is an important consideration in the future development of brain stimulation for therapeutic applications. 


\section{Acknowledgements and Funding}

The authors thank members of the Frohlich Lab for support and Christine Leddy for help with test administration. We thank Dr. Eric Youngstrom for help with the study design.

This work was supported by UNC Psychiatry, UNC School of Medicine, and the Swiss National Science Foundation (funding for CL, grant P2EZP3-152214). The electric field modeling work was supported in part by NIH grant R01MH091083. Research reported in this publication was partially supported by the National Institute of Mental Health of the National Institutes of Health under Award Number

R01MH101547. The content is solely the responsibility of the authors and does not necessarily represent the official views of the National Institutes of Health. 


\section{References}

[1] Prabhakaran V, Smith JA, Desmond JE, Glover GH, Gabrieli JD. Neural substrates of fluid reasoning: an $\mathrm{fMRI}$ study of neocortical activation during performance of the Raven's Progressive Matrices Test. Cognitive psychology. 1997;33:43-63.

[2] Esposito G, Kirkby BS, Van Horn JD, Ellmore TM, Berman KF. Context-dependent, neural systemspecific neurophysiological concomitants of ageing: mapping PET correlates during cognitive activation. Brain : a journal of neurology. 1999;122 ( Pt 5):963-79.

[3] Duncan J, Seitz RJ, Kolodny J, Bor D, Herzog H, Ahmed A, et al. A neural basis for general intelligence. Science. 2000;289:457-60.

[4] Duncan J, Owen AM. Common regions of the human frontal lobe recruited by diverse cognitive demands. Trends in neurosciences. 2000;23:475-83.

[5] Liu J, Zhang M, Jou J, Wu X, Li W, Qiu J. Neural bases of falsification in conditional proposition testing: evidence from an fMRI study. International journal of psychophysiology : official journal of the International Organization of Psychophysiology. 2012;85:249-56.

[6] Bunge SA, Ochsner KN, Desmond JE, Glover GH, Gabrieli JD. Prefrontal regions involved in keeping information in and out of mind. Brain : a journal of neurology. 2001;124:2074-86.

[7] Krawczyk DC, Michelle McClelland M, Donovan CM. A hierarchy for relational reasoning in the prefrontal cortex. Cortex; a journal devoted to the study of the nervous system and behavior. 2011;47:588-97.

[8] Nagahama Y, Okada T, Katsumi Y, Hayashi T, Yamauchi H, Sawamoto N, et al. Transient neural activity in the medial superior frontal gyrus and precuneus time locked with attention shift between object features. Neurolmage. 1999;10:193-9.

[9] Hampson M, Driesen NR, Skudlarski P, Gore JC, Constable RT. Brain connectivity related to working memory performance. The Journal of neuroscience : the official journal of the Society for Neuroscience. 2006;26:13338-43.

[10] Raichle ME, MacLeod AM, Snyder AZ, Powers WJ, Gusnard DA, Shulman GL. A default mode of brain function. Proceedings of the National Academy of Sciences of the United States of America. 2001;98:676-82.

[11] Shulman GL, Fiez JA, Corbetta M, Buckner RL, Miezin FM, Raichle ME, et al. Common Blood Flow Changes across Visual Tasks: II. Decreases in Cerebral Cortex. Journal of cognitive neuroscience. 1997;9:648-63.

[12] du Boisgueheneuc F, Levy R, Volle E, Seassau M, Duffau H, Kinkingnehun S, et al. Functions of the left superior frontal gyrus in humans: a lesion study. Brain : a journal of neurology. 2006;129:3315-28.

[13] Gray JR, Chabris CF, Braver TS. Neural mechanisms of general fluid intelligence. Nature neuroscience. 2003;6:316-22.

[14] Cohen Kadosh R. Modulating and enhancing cognition using brain stimulation: Science and fiction. Journal of Cognitive Psychology. 2015;27:141-63.

[15] Tremblay S, Lepage JF, Latulipe-Loiselle A, Fregni F, Pascual-Leone A, Theoret $\mathrm{H}$. The uncertain outcome of prefrontal tDCS. Brain stimulation. 2014;7:773-83.

[16] Horvath J, Forte C, O C. Quantitative Review Finds No Evidence of Cognitive Effects in Healthy Populations From Single-session Transcranial Direct Current Stimulation (tDCS). Brain stimulation. 2015. [17] Purpura DP, McMurtry JG. Intracellular Activities and Evoked Potential Changes during Polarization of Motor Cortex. Journal of neurophysiology. 1965;28:166-85.

[18] Nitsche MA, Paulus W. Excitability changes induced in the human motor cortex by weak transcranial direct current stimulation. The Journal of physiology. 2000;527 Pt 3:633-9. 
[19] Ali MM, Sellers KK, Frohlich F. Transcranial alternating current stimulation modulates large-scale cortical network activity by network resonance. The Journal of neuroscience : the official journal of the Society for Neuroscience. 2013;33:11262-75.

[20] Frohlich F, McCormick DA. Endogenous electric fields may guide neocortical network activity. Neuron. 2010;67:129-43.

[21] Reato D, Rahman A, Bikson M, Parra LC. Low-intensity electrical stimulation affects network dynamics by modulating population rate and spike timing. The Journal of neuroscience : the official journal of the Society for Neuroscience. 2010;30:15067-79.

[22] Ozen S, Sirota A, Belluscio MA, Anastassiou CA, Stark E, Koch C, et al. Transcranial electric stimulation entrains cortical neuronal populations in rats. The Journal of neuroscience : the official journal of the Society for Neuroscience. 2010;30:11476-85.

[23] Dayan E, Censor N, Buch ER, Sandrini M, Cohen LG. Noninvasive brain stimulation: from physiology to network dynamics and back. Nature neuroscience. 2013;16:838-44.

[24] Fritsch B, Reis J, Martinowich K, Schambra HM, Ji Y, Cohen LG, et al. Direct current stimulation promotes BDNF-dependent synaptic plasticity: potential implications for motor learning. Neuron. 2010;66:198-204.

[25] Antal A, Chaieb L, Moliadze V, Monte-Silva K, Poreisz C, Thirugnanasambandam N, et al. Brainderived neurotrophic factor (BDNF) gene polymorphisms shape cortical plasticity in humans. Brain stimulation. 2010;3:230-7.

[26] Chaieb L, Antal A, Ambrus GG, Paulus W. Brain-derived neurotrophic factor: its impact upon neuroplasticity and neuroplasticity inducing transcranial brain stimulation protocols. Neurogenetics. 2014;15:1-11.

[27] Glascher J, Tranel D, Paul LK, Rudrauf D, Rorden C, Hornaday A, et al. Lesion mapping of cognitive abilities linked to intelligence. Neuron. 2009;61:681-91.

[28] Kaufman A, Lichtenberger E. Assessing Adolescent and Adult Intelligence. 3rd ed. Hoboken, NJ: Wiley; 2005.

[29] Wechsler D. Wechsler Adult Intellignece Scale: Technical and Interpretive Manual. 4th ed. San Antonio, TX, USA: Pearson Education, Inc; 2008.

[30] Takeuchi H, Taki Y, Kawashima R. Effects of working memory training on cognitive functions and neural systems. Reviews in the neurosciences. 2010;21:427-49.

[31] Salthouse TA, Pink JE. Why is working memory related to fluid intelligence? Psychonomic bulletin \& review. 2008;15:364-71.

[32] Unsworth N, Engle RW. On the division of short-term and working memory: an examination of simple and complex span and their relation to higher order abilities. Psychological bulletin. 2007;133:1038-66.

[33] Groth-Marnat G. Handbook of Psychological Assessment. 4th ed. Hoboken, NJ: John Wiley \& Sons; 2003.

[34] Sattler J. Assessment of Children: Cognitive Foundations. 5th ed: Jerome M. Sattler, Publisher; 2008.

[35] Sattler J. Resource Guide to Accompany Assessment of Children: Cognitive Foundations. 5th ed: Jerome M. Sattler Publisher; 2008.

[36] Sattler J, Ryan J. Assessment with the WAIS-IV. La Mesa, CA: Jerone M Sattler Publisher; 2009.

[37] Wechsler D. Wechsler Adult Intelligence Scale - Fourth Edition: Administration and Scoring Manual.

San Antonio, TX: Pearson Education, Inc.; 2008.

[38] Bikson M, Datta A, Elwassif M. Establishing safety limits for transcranial direct current stimulation. Clinical neurophysiology : official journal of the International Federation of Clinical Neurophysiology. 2009;120:1033-4.

[39] Poreisz C, Boros K, Antal A, Paulus W. Safety aspects of transcranial direct current stimulation concerning healthy subjects and patients. Brain research bulletin. 2007;72:208-14. 
[40] Lee WH, Deng ZD, Kim TS, Laine AF, Lisanby SH, Peterchev AV. Regional electric field induced by electroconvulsive therapy in a realistic finite element head model: influence of white matter anisotropic conductivity. Neurolmage. 2012;59:2110-23.

[41] Lee WH, Lisanby SH, Laine AF, Peterchev AV. Stimulation strength and focality of electroconvulsive therapy and magnetic seizure therapy in a realistic head model. IEEE Eng Med Biol Conf 2014. 2014; In press.

[42] Zhang $Y$, Brady M, Smith S. Segmentation of brain MR images through a hidden Markov random field model and the expectation-maximization algorithm. IEEE transactions on medical imaging. 2001;20:45-57.

[43] Yushkevich PA, Piven J, Hazlett HC, Smith RG, Ho S, Gee JC, et al. User-guided 3D active contour segmentation of anatomical structures: significantly improved efficiency and reliability. Neurolmage. 2006;31:1116-28.

[44] Pons JP, Segonne E, Boissonnat JD, Rineau L, Yvinec M, Keriven R. High-quality consistent meshing of multi-label datasets. Information processing in medical imaging : proceedings of the conference. 2007;20:198-210.

[45] Team RC. R: A language and environment for statistical computing. . Vienna, Austria: R Foundation for Statistical Computing; 2014.

[46] Bates D, Maechler M, Bolker B, Walker S. Ime4: Linear mixed-effects models using Eigen and S4. R package version 1.1-6. 2014.

[47] Halekoh U, Hojsgaard S. pbkrtest: Parametric bootstrap and Kenward Roger based methods for mixed model comparison. R package version 0.3-8. 2013.

[48] Halekoh U, Hojsgaard S. A Kenward-Roger Approximation and Parametric Bootstrap Methods for Tests in Linear Mixed Models - the R Package pbkrtest. Journal of Statistical Software.Submitted.

[49] Batsikadze G, Moliadze V, Paulus W, Kuo MF, Nitsche MA. Partially non-linear stimulation intensitydependent effects of direct current stimulation on motor cortex excitability in humans. The Journal of physiology. 2013;591:1987-2000.

[50] Fathi D, Ueki Y, Mima T, Koganemaru S, Nagamine T, Tawfik A, et al. Effects of aging on the human motor cortical plasticity studied by paired associative stimulation. Clinical neurophysiology : official journal of the International Federation of Clinical Neurophysiology. 2010;121:90-3.

[51] Snowball A, Tachtsidis I, Popescu T, Thompson J, Delazer M, Zamarian L, et al. Long-term enhancement of brain function and cognition using cognitive training and brain stimulation. Current biology : CB. 2013;23:987-92.

[52] Nord CL, Lally N, Charpentier CJ. Harnessing electric potential: DLPFC tDCS induces widespread brain perfusion changes. Frontiers in systems neuroscience. 2013;7:99.

[53] Stagg CJ, Nitsche MA. Physiological basis of transcranial direct current stimulation. The Neuroscientist : a review journal bringing neurobiology, neurology and psychiatry. 2011;17:37-53. [54] Nitsche MA, Fricke K, Henschke U, Schlitterlau A, Liebetanz D, Lang N, et al. Pharmacological modulation of cortical excitability shifts induced by transcranial direct current stimulation in humans. The Journal of physiology. 2003;553:293-301.

[55] Liebetanz D, Nitsche MA, Tergau F, Paulus W. Pharmacological approach to the mechanisms of transcranial DC-stimulation-induced after-effects of human motor cortex excitability. Brain : a journal of neurology. 2002;125:2238-47.

[56] Jacobson L, Koslowsky M, Lavidor M. tDCS polarity effects in motor and cognitive domains: a metaanalytical review. Experimental brain research. 2012;216:1-10.

[57] Stagg CJ, Lin RL, Mezue M, Segerdahl A, Kong Y, Xie J, et al. Widespread modulation of cerebral perfusion induced during and after transcranial direct current stimulation applied to the left dorsolateral prefrontal cortex. The Journal of neuroscience : the official journal of the Society for Neuroscience. 2013;33:11425-31. 
[58] Keeser D, Meindl T, Bor J, Palm U, Pogarell O, Mulert C, et al. Prefrontal transcranial direct current stimulation changes connectivity of resting-state networks during fMRI. The Journal of neuroscience : the official journal of the Society for Neuroscience. 2011;31:15284-93.

[59] Falcone B, Coffman BA, Clark VP, Parasuraman R. Transcranial direct current stimulation augments perceptual sensitivity and 24-hour retention in a complex threat detection task. PloS one.

2012;7:e34993.

[60] Clark VP, Coffman BA, Mayer AR, Weisend MP, Lane TD, Calhoun VD, et al. TDCS guided using fMRI significantly accelerates learning to identify concealed objects. Neurolmage. 2012;59:117-28.

[61] Sehm B, Schnitzler T, Obleser J, Groba A, Ragert P, Villringer A, et al. Facilitation of inferior frontal cortex by transcranial direct current stimulation induces perceptual learning of severely degraded speech. The Journal of neuroscience : the official journal of the Society for Neuroscience.

2013;33:15868-78.

[62] Pirulli C, Fertonani A, Miniussi C. The role of timing in the induction of neuromodulation in perceptual learning by transcranial electric stimulation. Brain stimulation. 2013;6:683-9.

[63] Reinhart RM, Woodman GF. Enhancing long-term memory with stimulation tunes visual attention in one trial. Proceedings of the National Academy of Sciences of the United States of America.

2015;112:625-30.

[64] Zmigrod S, Colzato LS, Hommel B. Evidence for a role of the right dorsolateral prefrontal cortex in controlling stimulus-response integration: a transcranial direct current stimulation (tDCS) study. Brain stimulation. 2014;7:516-20.

[65] Zmigrod S. The role of the parietal cortex in multisensory and response integration: evidence from transcranial direct current stimulation (tDCS). Multisensory research. 2014;27:161-72.

[66] Reinhart RM, Woodman GF. Causal control of medial-frontal cortex governs electrophysiological and behavioral indices of performance monitoring and learning. The Journal of neuroscience : the official journal of the Society for Neuroscience. 2014;34:4214-27.

[67] Peters MA, Thompson B, Merabet LB, Wu AD, Shams L. Anodal tDCS to V1 blocks visual perceptual learning consolidation. Neuropsychologia. 2013;51:1234-9.

[68] Keeser D, Padberg F, Reisinger E, Pogarell O, Kirsch V, Palm U, et al. Prefrontal direct current stimulation modulates resting EEG and event-related potentials in healthy subjects: a standardized low resolution tomography (sLORETA) study. Neurolmage. 2011;55:644-57.

[69] Ohn SH, Park Cl, Yoo WK, Ko MH, Choi KP, Kim GM, et al. Time-dependent effect of transcranial direct current stimulation on the enhancement of working memory. Neuroreport. 2008;19:43-7.

[70] Meiron O, Lavidor M. Unilateral prefrontal direct current stimulation effects are modulated by working memory load and gender. Brain stimulation. 2013;6:440-7.

[71] Berryhill ME, Jones KT. tDCS selectively improves working memory in older adults with more education. Neuroscience letters. 2012;521:148-51.

[72] Lally N, Nord CL, Walsh V, Roiser JP. Does excitatory fronto-extracerebral tDCS lead to improved working memory performance? F1000Research. 2013;2:219.

[73] Zaehle T, Sandmann P, Thorne JD, Jancke L, Herrmann CS. Transcranial direct current stimulation of the prefrontal cortex modulates working memory performance: combined behavioural and electrophysiological evidence. BMC neuroscience. 2011;12:2.

[74] Mylius V, Jung M, Menzler K, Haag A, Khader PH, Oertel WH, et al. Effects of transcranial direct current stimulation on pain perception and working memory. European journal of pain. 2012;16:974-82.

[75] Teo F, Hoy KE, Daskalakis ZJ, Fitzgerald PB. Investigating the Role of Current Strength in tDCS Modulation of Working Memory Performance in Healthy Controls. Frontiers in psychiatry. 2011;2:45. [76] Hoy KE, Emonson MR, Arnold SL, Thomson RH, Daskalakis ZJ, Fitzgerald PB. Testing the limits: Investigating the effect of tDCS dose on working memory enhancement in healthy controls. Neuropsychologia. 2013;51:1777-84. 
[77] Mulquiney PG, Hoy KE, Daskalakis ZJ, Fitzgerald PB. Improving working memory: exploring the effect of transcranial random noise stimulation and transcranial direct current stimulation on the dorsolateral prefrontal cortex. Clinical neurophysiology : official journal of the International Federation of Clinical Neurophysiology. 2011;122:2384-9.

[78] Fregni F, Boggio PS, Nitsche M, Bermpohl F, Antal A, Feredoes E, et al. Anodal transcranial direct current stimulation of prefrontal cortex enhances working memory. Experimental brain research. 2005;166:23-30.

[79] Jeon SY, Han SJ. Improvement of the working memory and naming by transcranial direct current stimulation. Annals of rehabilitation medicine. 2012;36:585-95.

[80] Andrews SC, Hoy KE, Enticott PG, Daskalakis ZJ, Fitzgerald PB. Improving working memory: the effect of combining cognitive activity and anodal transcranial direct current stimulation to the left dorsolateral prefrontal cortex. Brain stimulation. 2011;4:84-9.

[81] Gladwin TE, den Uyl TE, Wiers RW. Anodal tDCS of dorsolateral prefontal cortex during an Implicit Association Test. Neuroscience letters. 2012;517:82-6.

[82] Gladwin TE, den Uyl TE, Fregni FF, Wiers RW. Enhancement of selective attention by tDCS: interaction with interference in a Sternberg task. Neuroscience letters. 2012;512:33-7.

[83] Brunoni AR, Vanderhasselt MA. Working memory improvement with non-invasive brain stimulation of the dorsolateral prefrontal cortex: a systematic review and meta-analysis. Brain and cognition. 2014;86:1-9.

[84] Kim JH, Kim DW, Chang WH, Kim YH, Kim K, Im CH. Inconsistent outcomes of transcranial direct current stimulation may originate from anatomical differences among individuals: electric field simulation using individual MRI data. Neuroscience letters. 2014;564:6-10.

[85] Frohlich F, Schmidt SL. Rational design of transcranial current stimulation (TCS) through mechanistic insights into cortical network dynamics. Frontiers in human neuroscience. 2013;7:804.

[86] Herrmann CS, Rach S, Neuling T, Struber D. Transcranial alternating current stimulation: a review of the underlying mechanisms and modulation of cognitive processes. Frontiers in human neuroscience. 2013;7:279.

[87] Miller EK, Buschman TJ. Cortical circuits for the control of attention. Current opinion in neurobiology. 2013;23:216-22.

[88] Siegel M, Warden MR, Miller EK. Phase-dependent neuronal coding of objects in short-term memory. Proceedings of the National Academy of Sciences of the United States of America.

2009;106:21341-6.

[89] Duzel E, Penny WD, Burgess N. Brain oscillations and memory. Current opinion in neurobiology. 2010;20:143-9.

[90] Ward LM. Synchronous neural oscillations and cognitive processes. Trends in cognitive sciences. 2003;7:553-9.

[91] Uhlhaas PJ, Haenschel C, Nikolic D, Singer W. The role of oscillations and synchrony in cortical networks and their putative relevance for the pathophysiology of schizophrenia. Schizophrenia bulletin. 2008;34:927-43.

[92] Santarnecchi E, Polizzotto NR, Godone M, Giovannelli F, Feurra M, Matzen L, et al. Frequencydependent enhancement of fluid intelligence induced by transcranial oscillatory potentials. Current biology : CB. 2013;23:1449-53.

[93] Pahor A, Jausovec N. The effects of theta transcranial alternating current stimulation (tACS) on fluid intelligence. International journal of psychophysiology : official journal of the International Organization of Psychophysiology. 2014;93:322-31.

[94] Barbey AK, Colom R, Paul EJ, Grafman J. Architecture of fluid intelligence and working memory revealed by lesion mapping. Brain structure \& function. 2014;219:485-94. 
[95] Spearman C. "General intelligence " objectively determined and measured. Am J Psychol. 1904;15:201-92.

[96] Barbey AK, Colom R, Solomon J, Krueger F, Forbes C, Grafman J. An integrative architecture for general intelligence and executive function revealed by lesion mapping. Brain : a journal of neurology. 2012;135:1154-64.

[97] Glascher J, Rudrauf D, Colom R, Paul LK, Tranel D, Damasio H, et al. Distributed neural system for general intelligence revealed by lesion mapping. Proceedings of the National Academy of Sciences of the United States of America. 2010;107:4705-9.

[98] Jung RE, Haier RJ. The Parieto-Frontal Integration Theory (P-FIT) of intelligence: converging neuroimaging evidence. The Behavioral and brain sciences. 2007;30:135-54; discussion 54-87.

[99] Colom R, Haier RJ, Head K, Alvarez-Linera J, Quiroga MA, Shih PC, et al. Gray matter correlates of fluid, crystallized, and spatial intelligence: Testing the P-FIT model. Intelligence. 2009;37:124-35. [100] Barbey AK, Colom R, Grafman J. Dorsolateral prefrontal contributions to human intelligence. Neuropsychologia. 2013;51:1361-9.

[101] Sternberg RJ, Kaufman SB. The Cambridge Handbook of Intelligence. Cambridge ; New York: Cambridge University Press; 2011.

[102] Shaw P, Greenstein D, Lerch J, Clasen L, Lenroot R, Gogtay N, et al. Intellectual ability and cortical development in children and adolescents. Nature. 2006;440:676-9.

[103] Thompson PM, Cannon TD, Narr KL, van Erp T, Poutanen VP, Huttunen M, et al. Genetic influences on brain structure. Nature neuroscience. 2001;4:1253-8.

[104] Haier RJ, Jung RE, Yeo RA, Head K, Alkire MT. Structural brain variation and general intelligence. Neurolmage. 2004;23:425-33.

[105] Colom R, Jung RE, Haier RJ. Distributed brain sites for the g-factor of intelligence. Neurolmage. 2006;31:1359-65.

[106] Choi YY, Shamosh NA, Cho SH, DeYoung CG, Lee MJ, Lee JM, et al. Multiple bases of human intelligence revealed by cortical thickness and neural activation. The Journal of neuroscience : the official journal of the Society for Neuroscience. 2008;28:10323-9.

[107] Hulshoff Pol HE, Schnack HG, Posthuma D, Mandl RC, Baare WF, van Oel C, et al. Genetic contributions to human brain morphology and intelligence. The Journal of neuroscience : the official journal of the Society for Neuroscience. 2006;26:10235-42.

[108] Cole MW, Yarkoni T, Repovs G, Anticevic A, Braver TS. Global connectivity of prefrontal cortex predicts cognitive control and intelligence. The Journal of neuroscience : the official journal of the Society for Neuroscience. 2012;32:8988-99.

[109] van den Heuvel MP, Stam CJ, Kahn RS, Hulshoff Pol HE. Efficiency of functional brain networks and intellectual performance. The Journal of neuroscience : the official journal of the Society for Neuroscience. 2009;29:7619-24.

[110] Duncan J. The structure of cognition: attentional episodes in mind and brain. Neuron. 2013;80:3550.

[111] Olesen PJ, Westerberg H, Klingberg T. Increased prefrontal and parietal activity after training of working memory. Nature neuroscience. 2004;7:75-9.

[112] Bikson M, Bestmann S, Edwards D. Neuroscience: transcranial devices are not playthings. Nature. 2013;501:167.

[113] Brain blast. Nature. 2013;498:271-2.

[114] Estevis E, Basso MR, Combs D. Effects of practice on the Wechsler Adult Intelligence Scale-IV across 3- and 6-month intervals. The Clinical neuropsychologist. 2012;26:239-54.

[115] Berryhill ME, Peterson DJ, Jones KT, Stephens JA. Hits and misses: leveraging tDCS to advance cognitive research. Frontiers in psychology. 2014;5:800. 


\section{Figure Legends}

Figure 1. tDCS administered over DLPFC. Two stimulators were used to deliver double-blinded unilateral or bilateral tDCS over DLPFC. In all stimulation conditions, three electrodes were placed, one each at F3, F4 (anodes, red electrodes), and Cz (cathode/return, blue electrode) (A). For bilateral tDCS, both stimulators delivered 20 minutes of $2 \mathrm{~mA}$ stimulation with a ramp up and ramp down of current (B). For sham tDCS, both stimulators administered 20 seconds of stimulation with a ramp up and ramp down of current, in order to mimic the sensations of stimulation (C). For right tDCS, 20 minutes of stimulation was delivered through the stimulator attached to electrodes $F 4$ and $C z$, while only the 20 seconds of sham stimulation were delivered through the stimulator attached to F3 and Cz (D). For left tDCS, 20 minutes of stimulation was delivered through the stimulator attached to $\mathrm{F} 3$ and $\mathrm{Cz}$, while the stimulator attached to $\mathrm{F} 4$ and $\mathrm{Cz}$ administered 20 seconds of stimulation (E).

Figure 2. Electric field modeling of tDCS. Anodal tDCS was administered bilaterally over frontal cortex (A$D)$, unilaterally on the left $(E-G)$, or unilaterally on the right $(H-J)$. Red electrodes represent anodes, blue electrodes represent cathodes, and purple electrodes represent stimulation electrodes which were attached, but only received sham stimulation $(A, E, H)$. For bilateral stimulation, the greatest magnitude of the electric field was mostly localized to areas underneath and between the electrodes ( $\mathrm{B}$, left panels: gray matter; right panels: white matter). For unilateral stimulation, electric field was mostly localized to the hemisphere targeted for stimulation ( $F$ and I, left panels: gray matter; right panels: white matter). The middle frontal gyri (green) and superior frontal gyri (blue) were the areas of frontal cortex with the highest amplitude electric field (D). Axial, coronal, and sagittal sections showing electric field induced by $\operatorname{tDCS}(D, G, J)$. 
Figure 3. Double-blind, randomized, placebo-controlled study design with a repeated-measure of IQ.

During Session 1, each participant was administered the Wechsler Adult Intelligence Scale, Fourth Edition (WAIS-IV). During Session 2, each participant received either sham stimulation or bilateral tDCS (A, substudy 1 ) or either right or left tDCS (B, substudy 2 ). The WAIS-IV was immediately administered following stimulation in order to assess stimulation-induced modulation in performance.

Figure 4. Unilateral and bilateral tDCS significantly decreased practice gains in the Full Scale IQ (FSIQ) compared to sham stimulation. The difference in FSIQ between sessions (Session 2 - Session 1) was calculated for each participant, and then averaged across participants. Group means of individual differences are plotted. Error bars show 1 SEM. * indicates significant at $p<0.05$.

Figure 5. The WAIS-IV provides four index scores which represent the major components of intelligence: (A) Verbal Comprehension Index (VCI), (B) Perceptual Reasoning Index (PRI), (C) Working Memory Index (WMI), and (D) Processing Speed Index (PSI). Right tDCS significantly decreased practice gains on the PRI of the WAIS-IV, while left tDCS and bilateral tDCS decreased practice gains on the PRI at trend level. Bars represent the group means of the individual differences of scores between Session 1 and Session 2 . Error bars show 1 SEM. * indicates significant at $p<0.05$.

Figure 6. Perceptual reasoning abilities are assessed by 3 subtests: Block Design (A), Matrix Reasoning (B), and Visual Puzzles (C). Differences between stimulation conditions were found in Matrix Reasoning, Visual Puzzles, and Picture Completion. For Matrix Reasoning, right and bilateral tDCS decreased practice gains, at trend level, compared to sham stimulation. Interestingly, practice gains in Matrix Reasoning were unchanged by left tDCS compared to sham stimulation. For Visual Puzzles, right and left stimulation tDCS significantly reduced practice gains compared to sham stimulation. Bars represent the 
group means of the differences (by participant) of scores between Session 1 and Session 2. Error bars show 1 SEM. * indicates significant at $p<0.05$.

Figure 7. Changes in performance on the subtests were not influenced by test order for substudy 1 (A, sham or bilateral tDCS) or substudy 2 (B, right or left tDCS). Group means of the individual differences of each subtest score between Session 1 and Session 2 are plotted in order of test administration. Error bars show 1 SEM. 

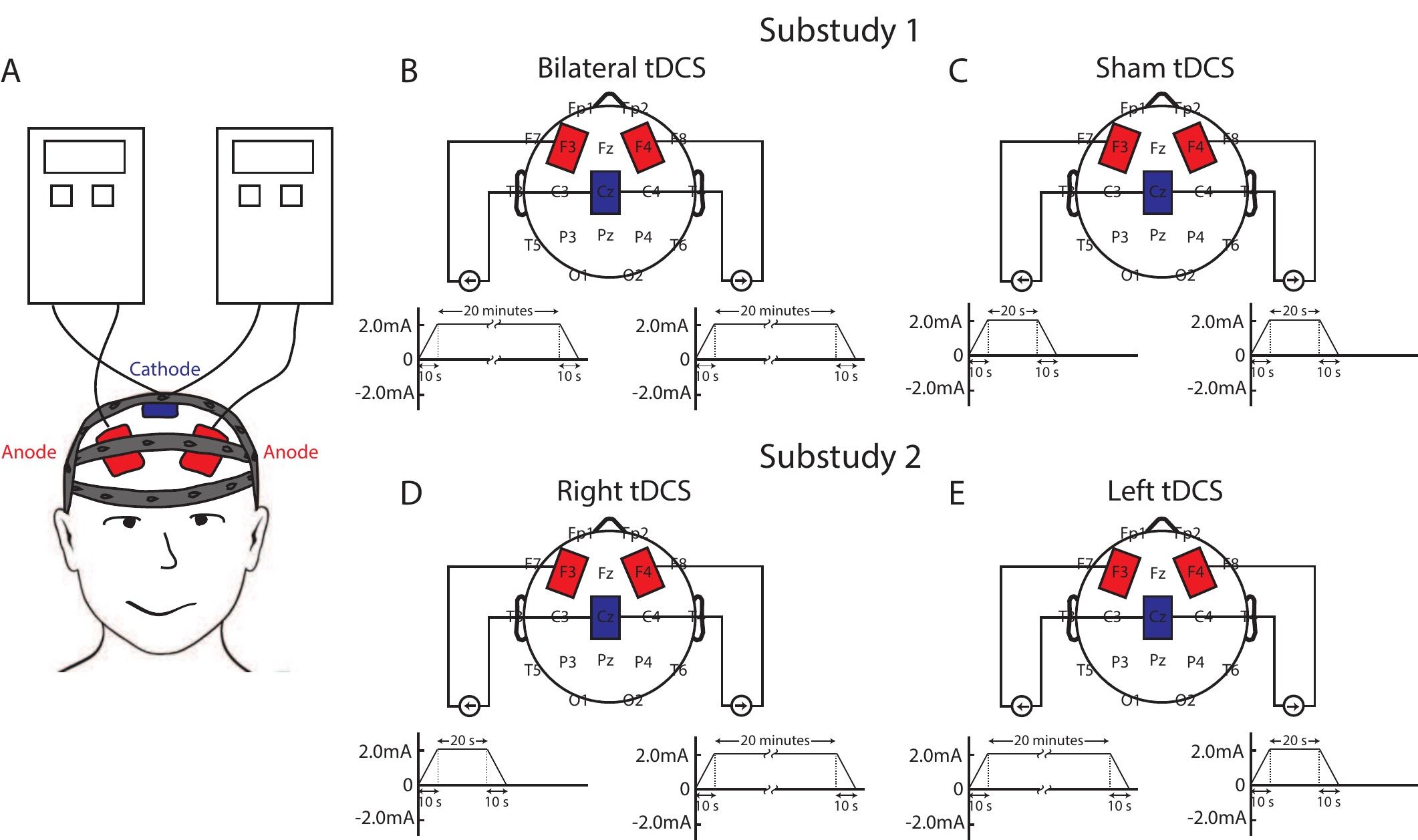
A

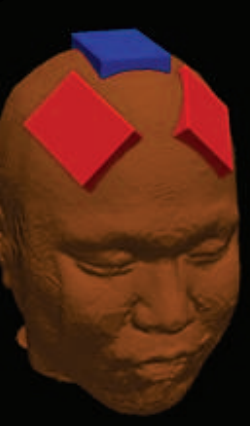

C

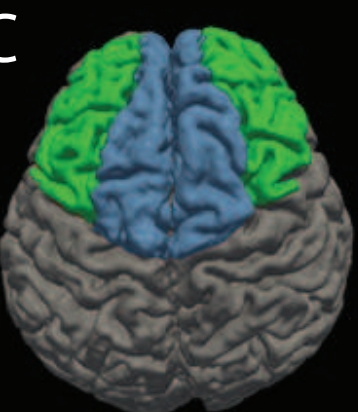

B

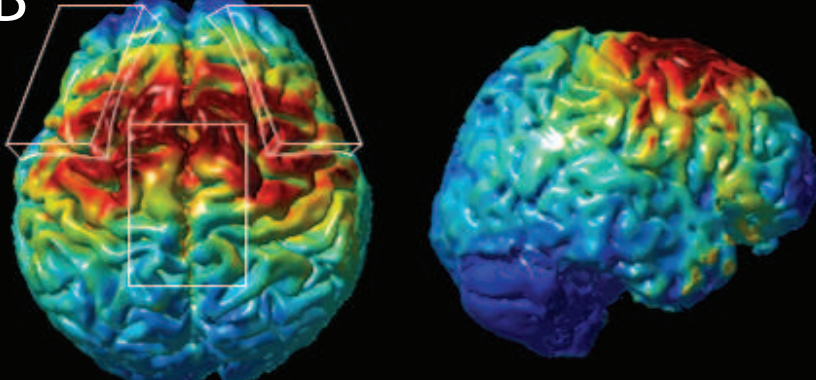

D

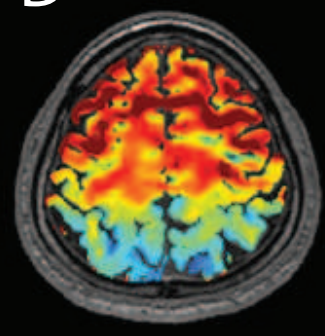

E

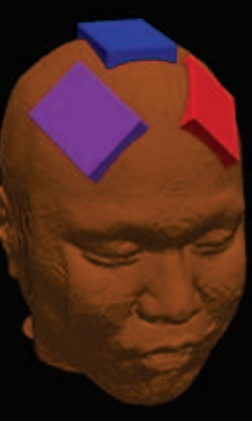

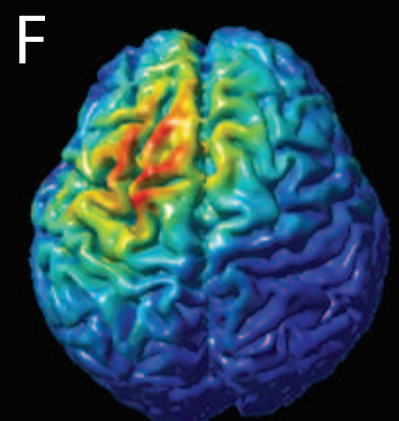

\section{$G$}

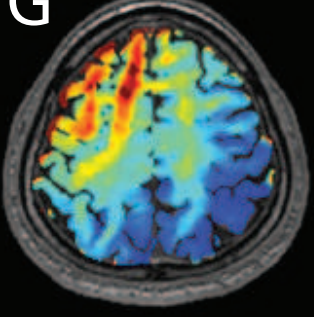

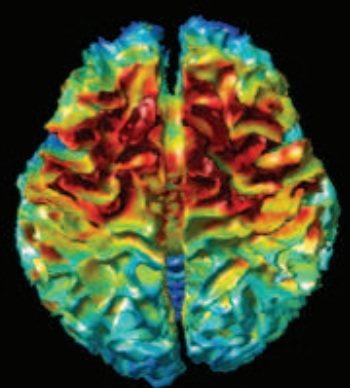
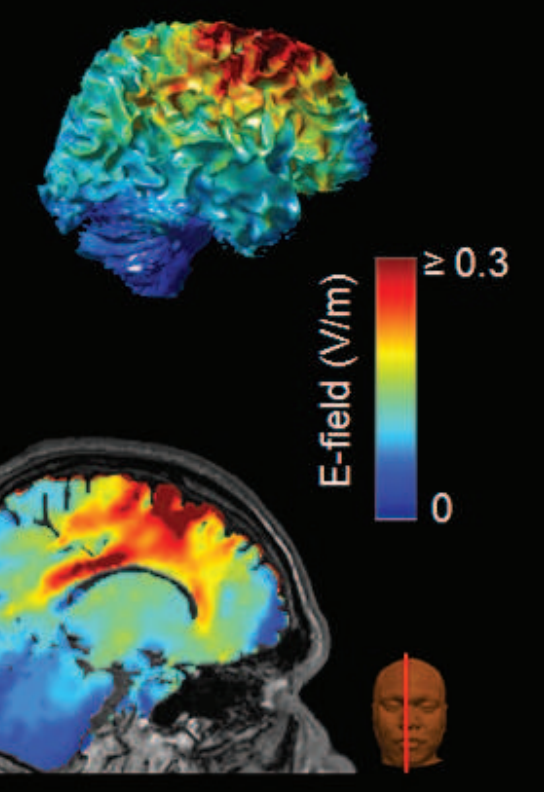

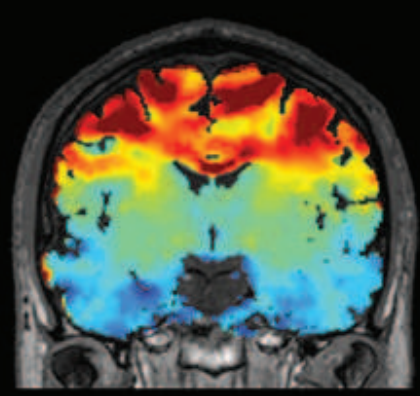

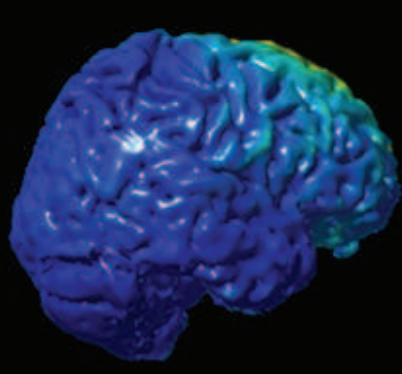
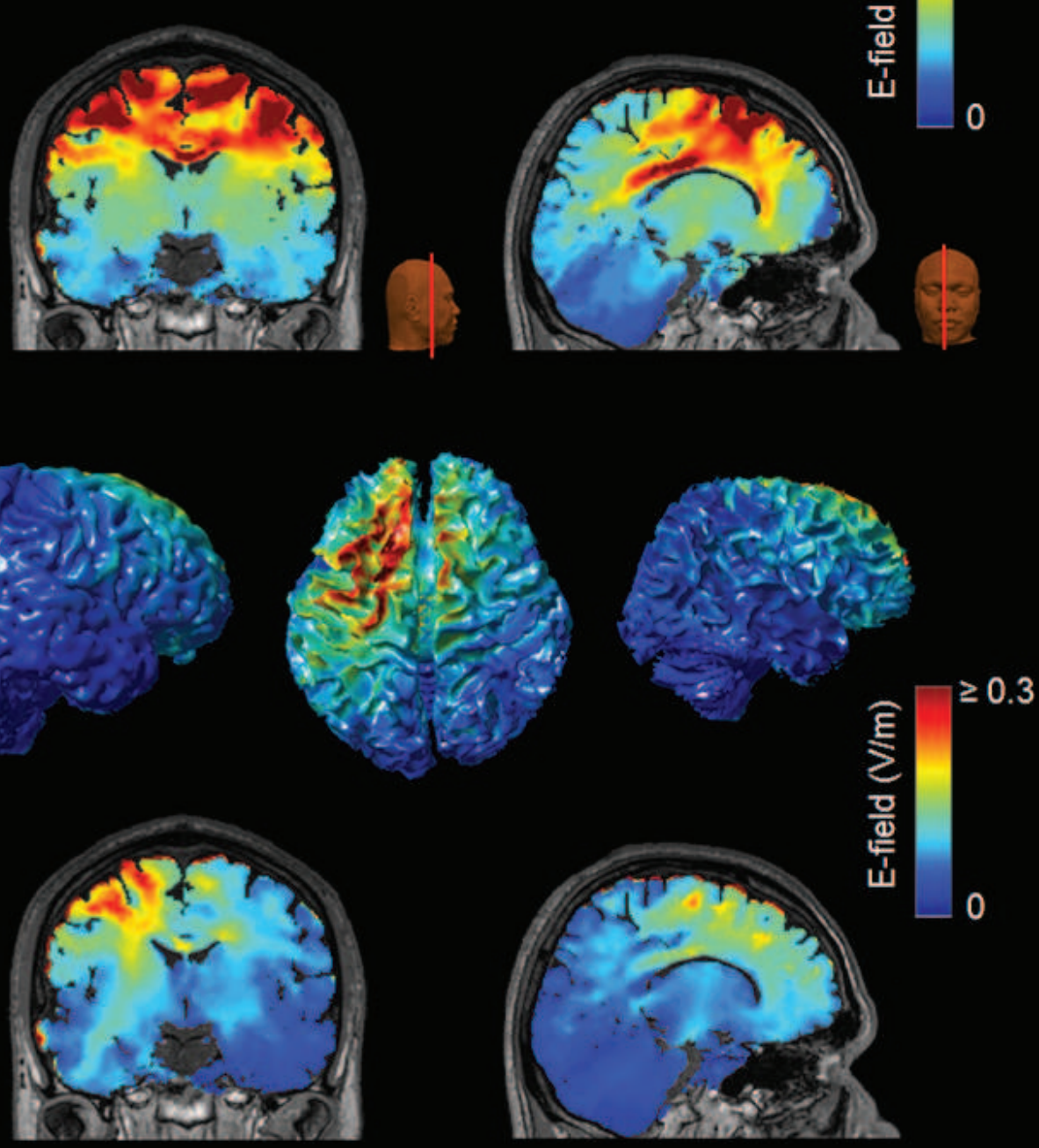

H
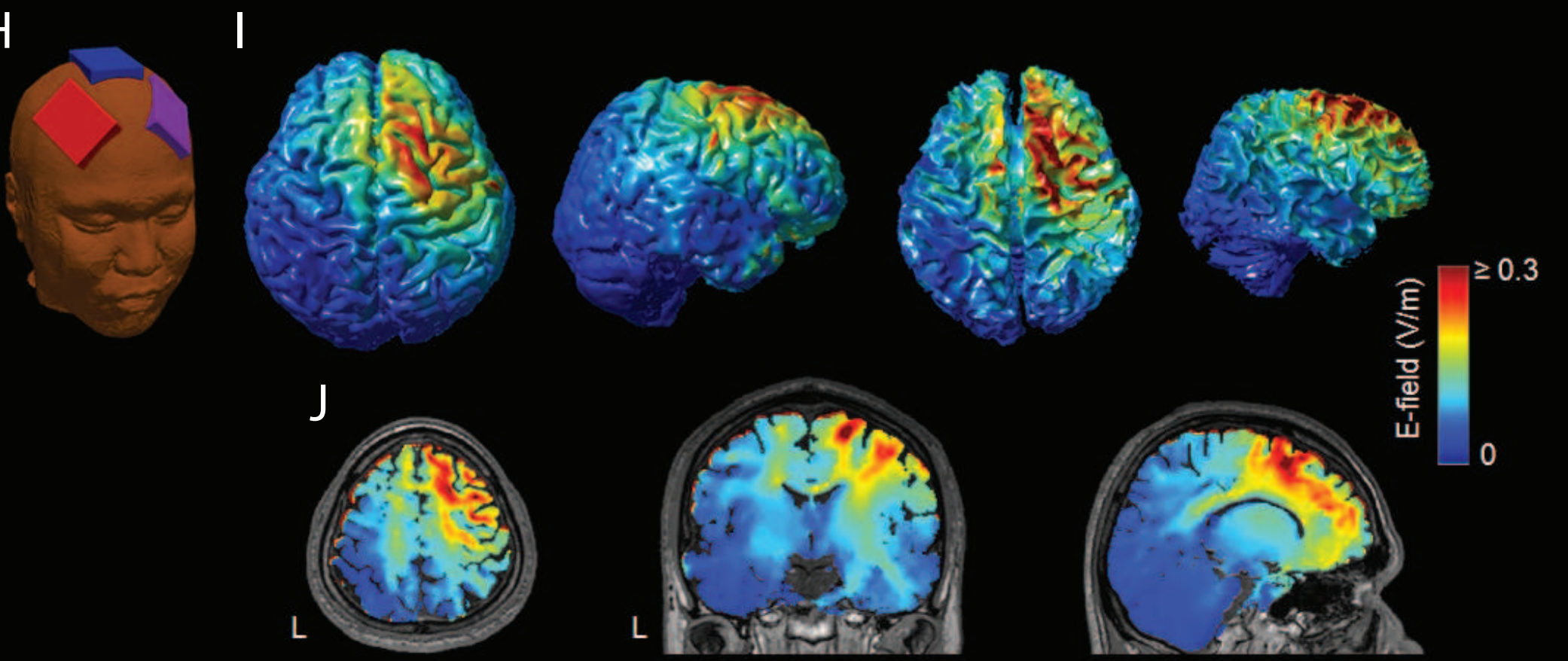

L

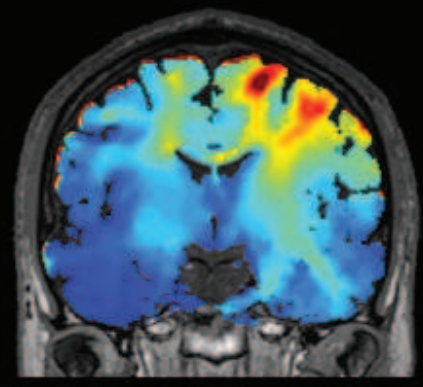


$A$

Session $1 \quad$ Session 2

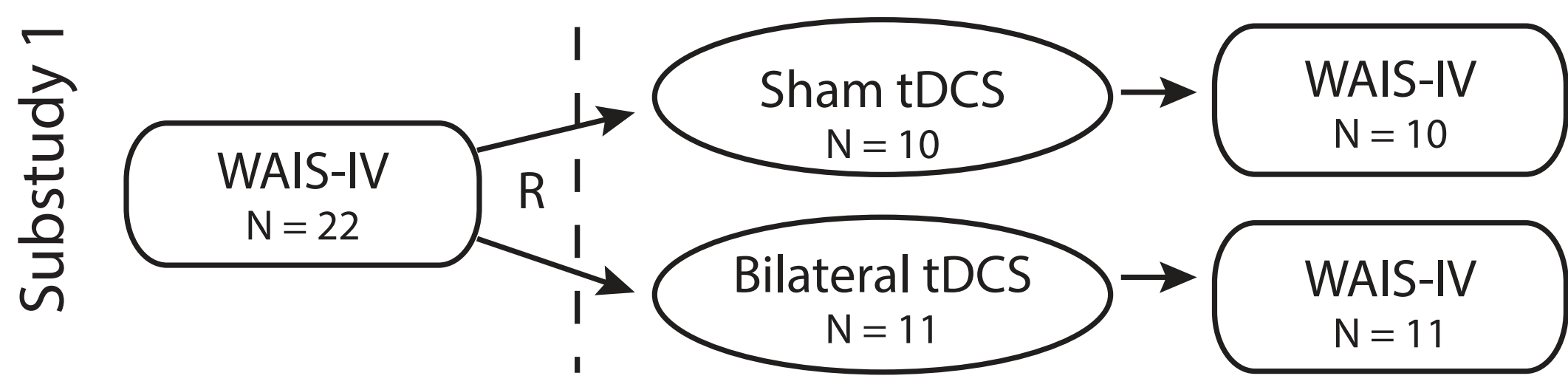

Double-Blind

B

Session 1

Session 2

$\frac{1}{2}$

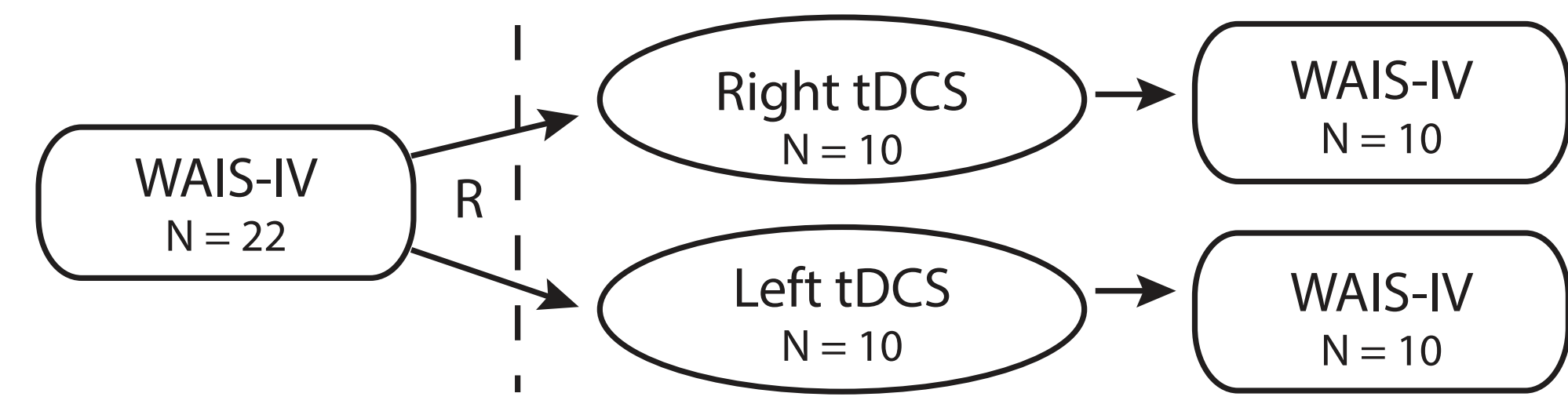

Double-Blind 
Figure 5
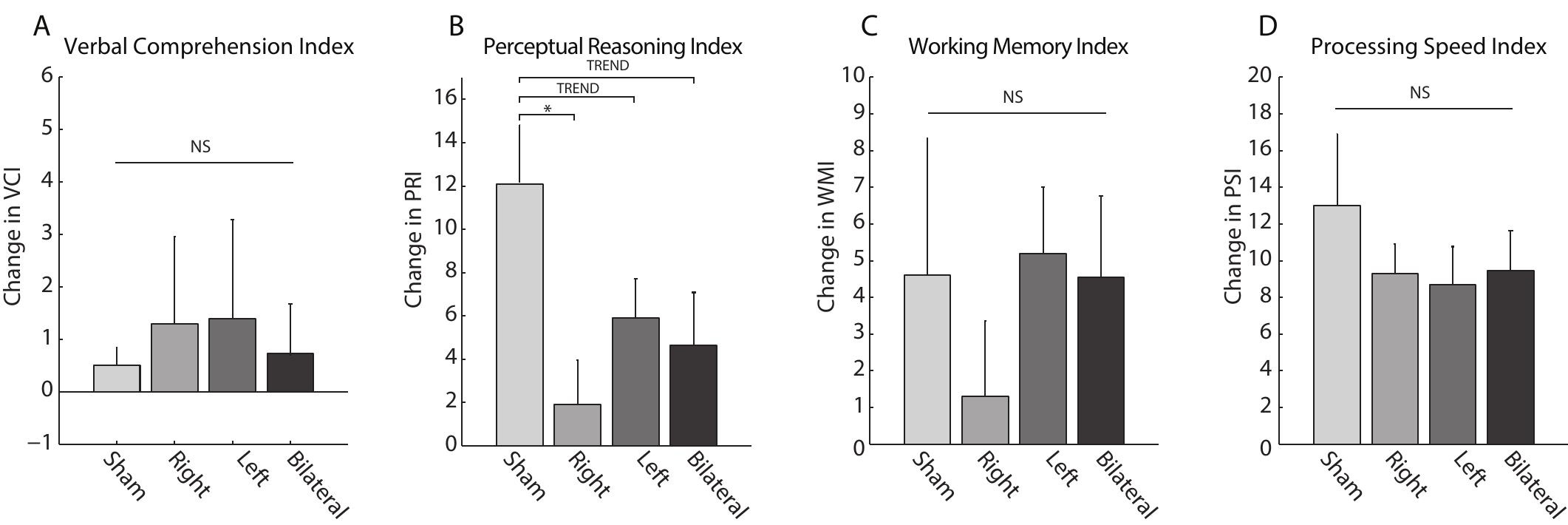
A

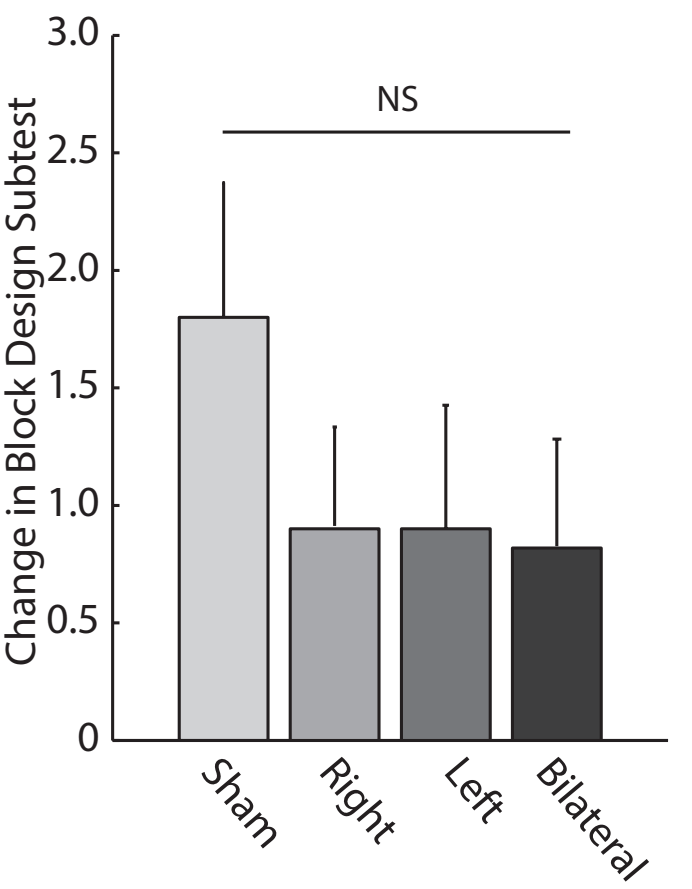

Block Design

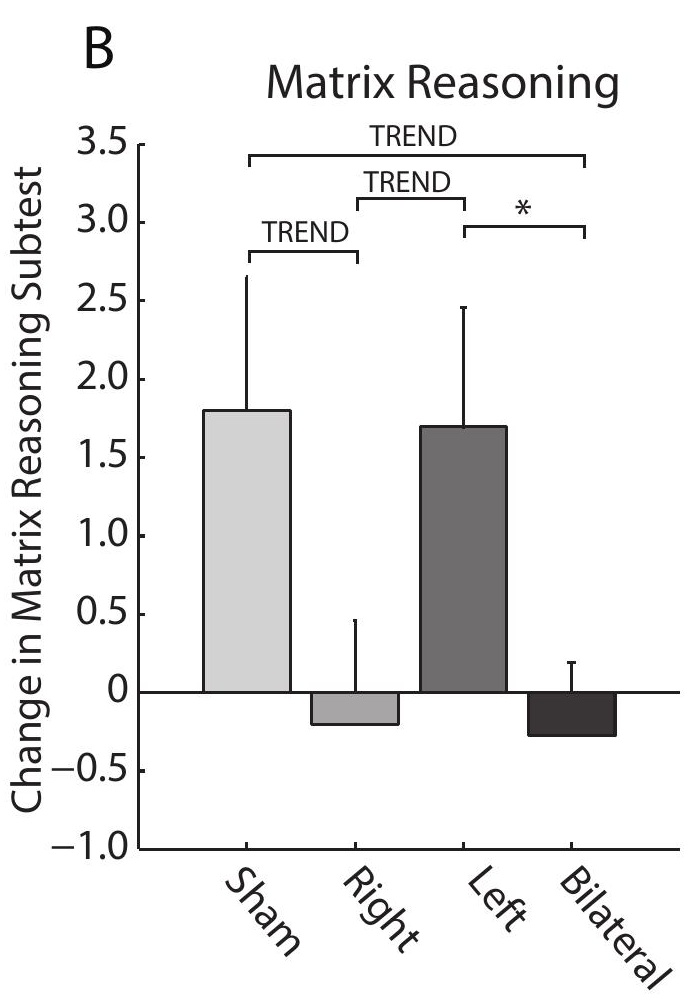

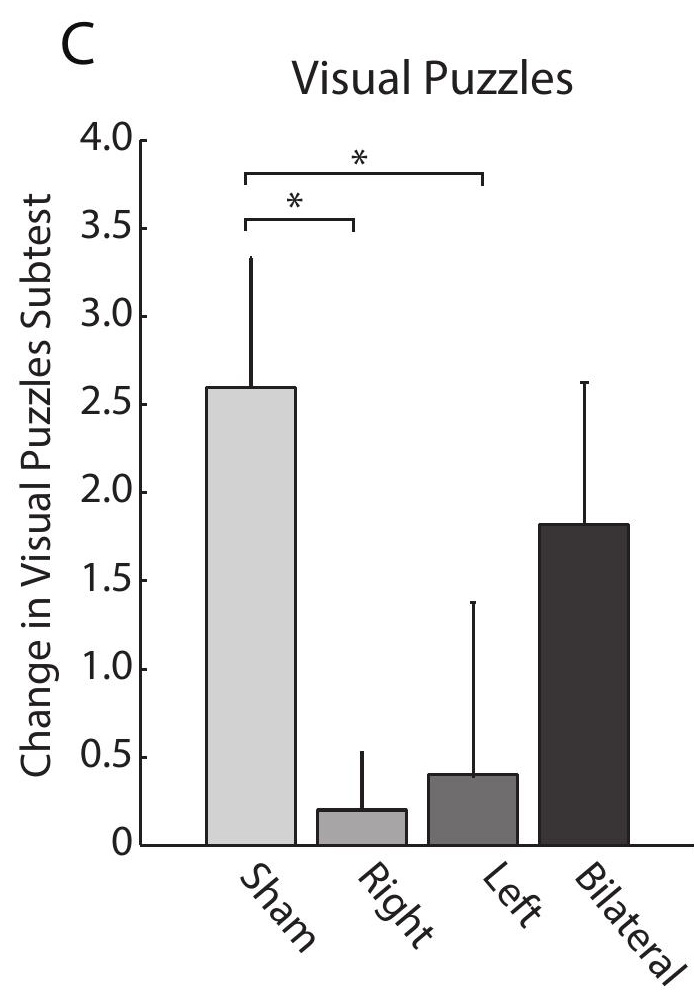




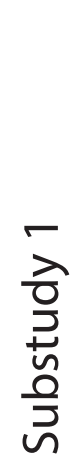

更

B

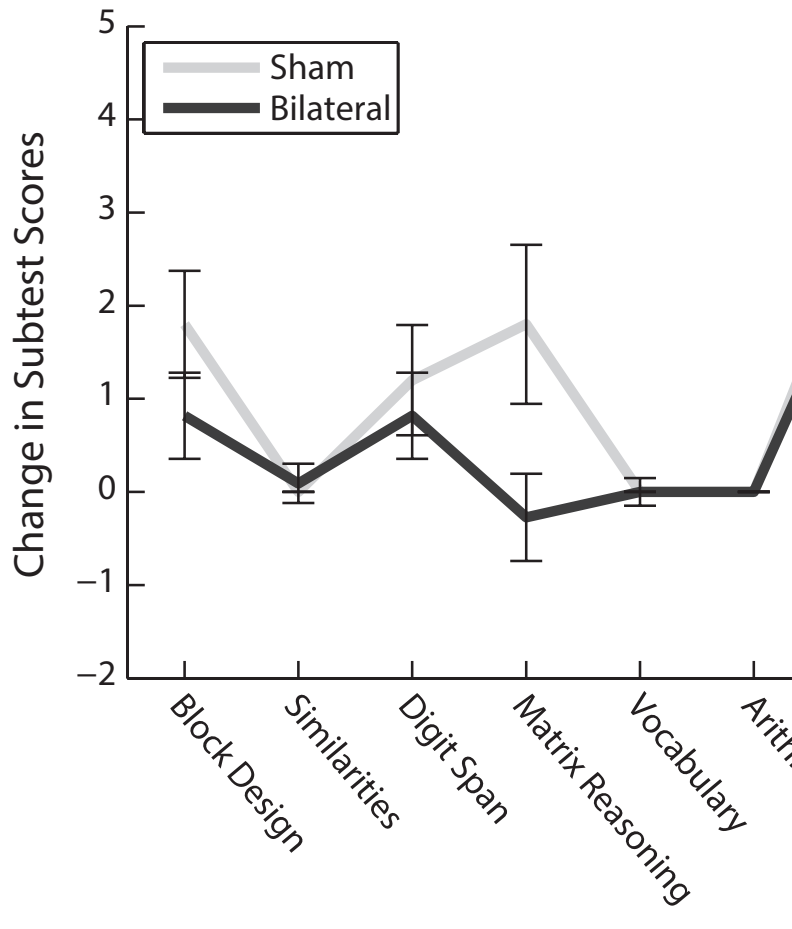

$\frac{1}{2}$
$\frac{1}{2}$
$\frac{0}{n}$

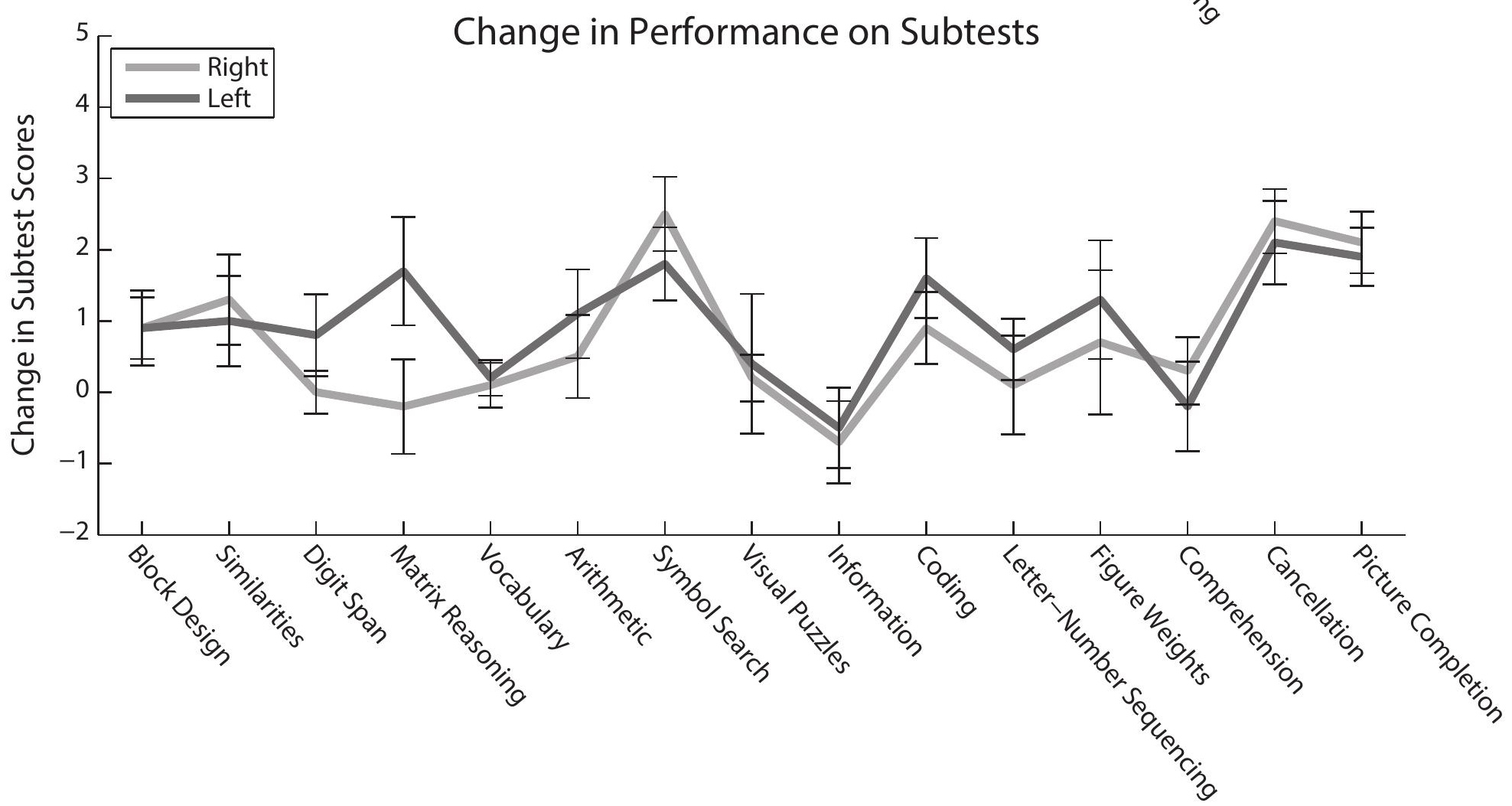


Table 1: WAIS-IV Indices and Subtests (according to test manual, Pearson Education, Inc.)

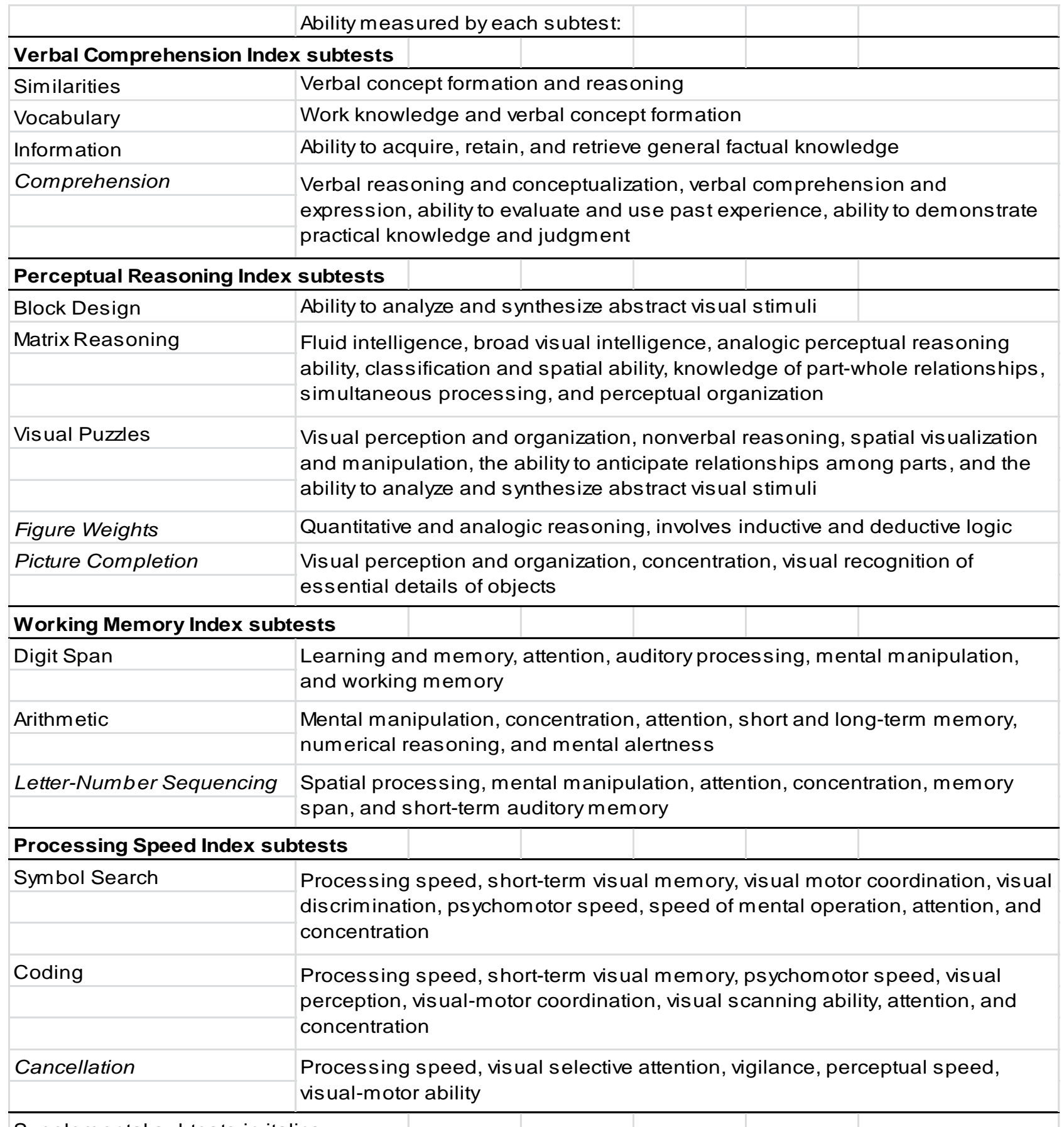

Supplemental subtests in italics 
Table 2: WAIS-IV scores by group and session

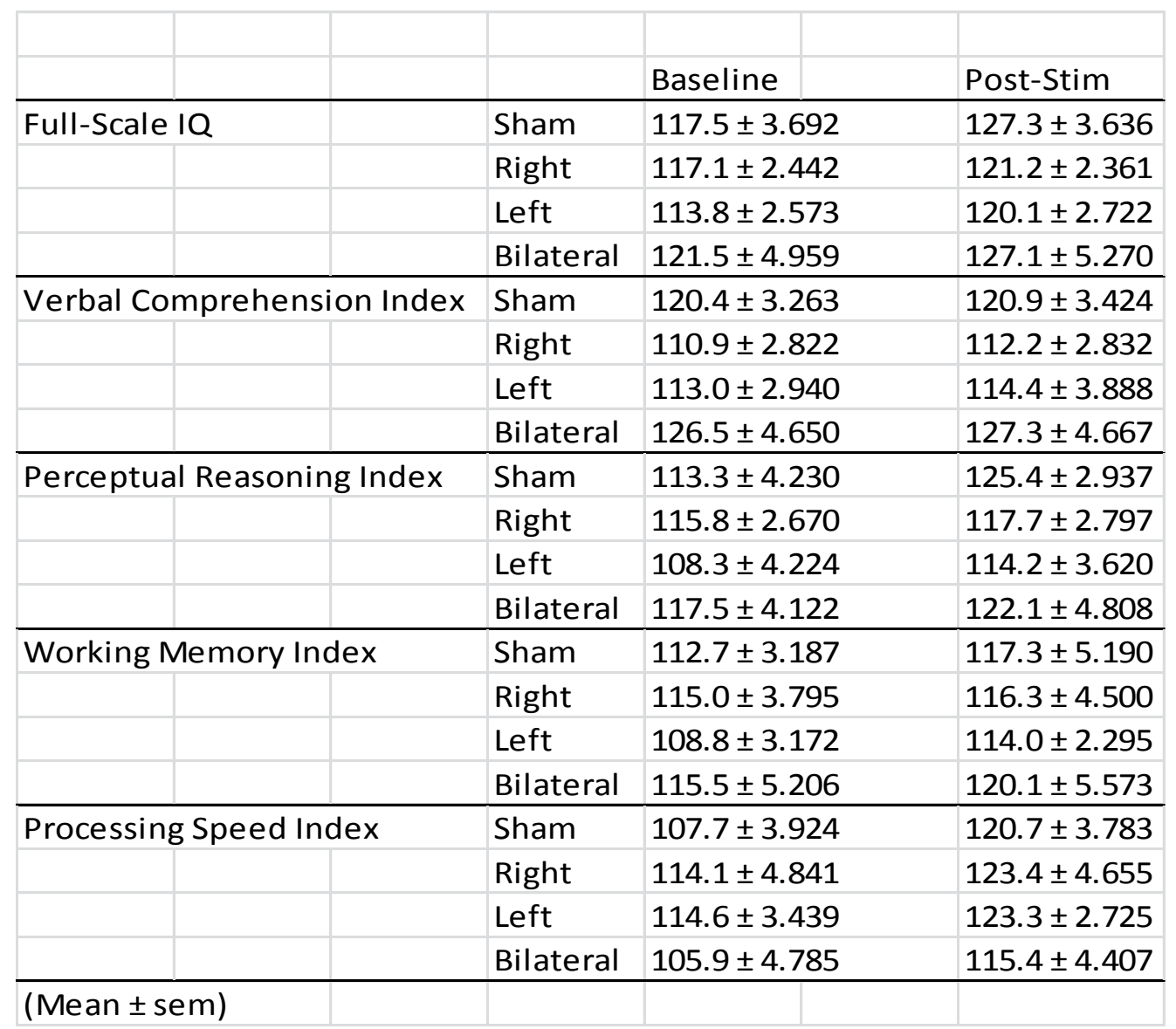

Table 3: Linear mixed model results for Full-Scale IQ (FSIQ) Index scales

\begin{tabular}{|c|c|c|c|c|c|c|}
\hline & \multicolumn{6}{|c|}{ Linear mixed models fixed factors } \\
\hline & \multicolumn{2}{|c|}{ Session } & \multicolumn{2}{|c|}{ Condition } & \multicolumn{2}{|c|}{ Session $\times$ Condition } \\
\hline \multicolumn{3}{|c|}{ Full-Scale IQ (FSIQ) Index Scales } & & & & \\
\hline $\mathrm{VCl}$ & $F(1,40)=2.29$ & $p>0.1$ & $F(3,37)=3.78$ & $p=0.0184^{* *}$ & $F(3,37)=0.106$ & $p>0.1$ \\
\hline PRI & $F(1,40)=23.8$ & $\mathrm{p}<0.001^{* *}$ & $F(3,37)=1.17$ & $p>0.1$ & $F(3,37)=3.45$ & $p=0.0261^{* *}$ \\
\hline WMI & $F(1,40)=9.90$ & $p=0.00312 * *$ & $F(3,37)=0.420$ & $p>0.1$ & $F(3,37)=0.471$ & $p>0.1$ \\
\hline PSI & $F(1,40)=63.9$ & $p<0.001 * *$ & $F(3,37)=1.04$ & $p>0.1$ & $F(3,37)=0.565$ & $p>0.1$ \\
\hline
\end{tabular}

** significant effects $(p<0.05)$, *trend-level effects $(p<0.1)$ 
Table 4: Linear mixed model results for Perceptual Reasoning Index (PRI) subtests

\begin{tabular}{|c|c|c|c|c|c|c|}
\hline & \multicolumn{6}{|c|}{ Linear mixed models fixed factors } \\
\hline & \multicolumn{2}{|c|}{ Session } & \multicolumn{2}{|c|}{ Condition } & \multicolumn{2}{|c|}{ Session $x$ Condition } \\
\hline \multicolumn{3}{|c|}{ Perceptual Reasoning Index (PRI) Subtests } & & & & \\
\hline Block Design & $F(1,40)=19.4$ & $p<0.001^{* *}$ & $F(3,37)=0.648$ & $p>0.1$ & $F(3,37)=0.853$ & $p>0.1$ \\
\hline Matrix Reasoning & $F(1,40)=3.95$ & $p=0.0536^{*}$ & $F(3,37)=0.750$ & $p>0.1$ & $F(3,37)=2.76$ & $p=0.0560^{*}$ \\
\hline Visual Puzzles & $F(1,40)=10.3$ & $p=0.00262 * *$ & $F(3,37)=3.41$ & $\mathrm{p}=0.0274 * *$ & $F(3,37)=2.28$ & $p=0.0949^{*}$ \\
\hline
\end{tabular}

** significant effects $(p<0.05), *$ trend-level effects $(p<0.1)$ 